Document downloaded from:

http://hdl.handle.net/10251/102317

This paper must be cited as:

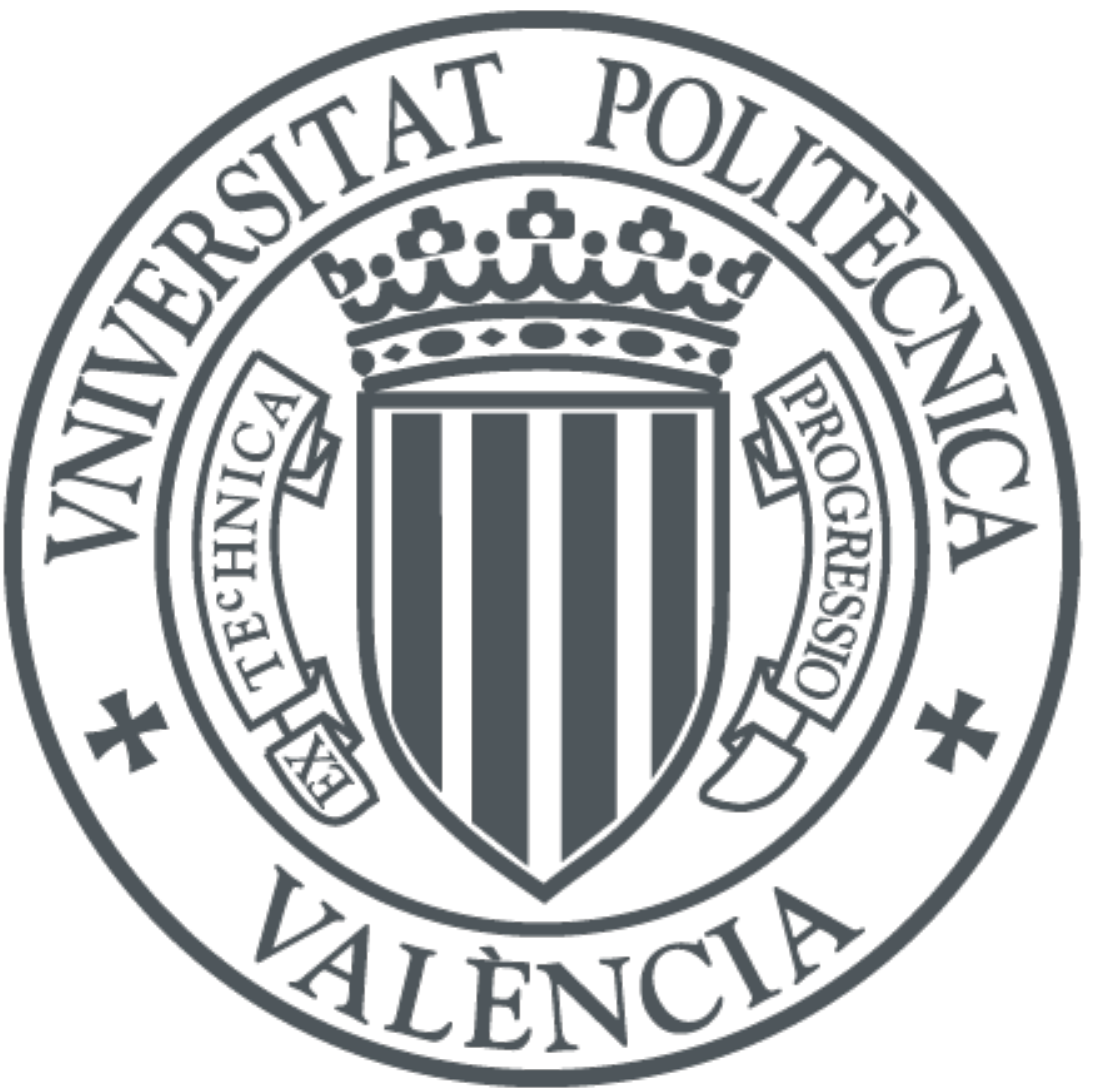

The final publication is available at

https://doi.org/10.1016/j.eswa.2017.03.028

Copyright Elsevier

Additional Information 


\section{A dynamic trading rule based on filtered flag pattern recognition for stock market price forecasting}

\section{Authors}

Rubén Arévalo

I.U. de Matemàtica Pura i Aplicada

Universitat Politècnica de València

Camí de Vera s/n, 46022, Valencia (Spain)

ruben1.6180@gmail.com

Jorge García

I.U. de Matemàtica Pura i Aplicada

Universitat Politècnica de València

Camí de Vera s/n, 46022, Valencia (Spain)

jorgeomar82@yahoo.es

Francisco Guijarro

I.U. de Matemàtica Pura i Aplicada

Universitat Politècnica de València

Camí de Vera s/n, 46022, Valencia (Spain)

fraguima@upvnet.upv.es

(*) Corresponding author

(+34) 963877000 (ext. 88204)

Alfred Peris

I.U. de Matemàtica Pura i Aplicada

Universitat Politècnica de València

Camí de Vera s/n, 46022, Valencia (Spain)

aperis@mat.upv.es 


\begin{abstract}
In this paper we propose and validate a trading rule based on flag pattern recognition, incorporating important innovations with respect to the previous research. Firstly, we propose a dynamic window scheme that allows the stop loss and take profit to be updated on a quarterly basis. In addition, since the flag pattern is a trend-following pattern, we have added the EMA indicator to filter trades. This technical analysis indicator is calculated both for 15-minute and 1-day timeframes, which enables short and medium terms to be considered simultaneously. We also filter the flags according to the price range on which they are developed and have limited the maximum loss of each trade to 100 points. The proposed methodology was applied to 91,309 intraday observations of the DJIA index, considerably improving the results obtained in the previous proposals and those obtained by the buy \& hold strategy, both for profitability and risk, and also after taking into account the transaction costs. These results seem to challenge market efficiency in line with other similar studies, in the specific analysis carried out on the DJIA index and is also limited to the setup considered.
\end{abstract}

\title{
Highlights
}

We propose an automatic and dynamic trading rule based on flag pattern recognition.

The strategy does not depend on the ability of the trader to guess the best configuration of the trading rule.

We include several filters for the trades, one of them considering the EMA indicator in short and medium timeframes.

The trading rule is applied on a large intraday database for the DJIA index.

We can conclude that our proposal is far superior to the previous flag pattern strategies as regards both profitability and risk.

\section{Keywords}

Financial markets; Technical analysis; Chart pattern; Exponential moving average; Trading system; Automatic trading

\section{Acknowledgements}

The fourth author of this work was partially supported by MINECO, Project MTM201675963-P. 


\section{Introduction}

Financial markets are complex and non-linear dynamic systems, so their predictions are really challenging (Barak, Dahooie, \& Tichý, 2015). Investment institutions, pension funds and private investors daily face the problem of investing billions of dollars in assets with the intention of achieving a high profit with a low risk. For these purposes professional investors use two major approaches to making accurate decisions in financial markets: fundamental analysis and technical analysis. Fundamental analysis uses macroeconomic, industrial and/or business variables to predict the stock value of firms, whereas technical analysis is based on past stock prices under the assumption that historical behavior has a testable effect on the future evolution of the stock price.

Technical analysis confronts the Efficient Market Hypothesis (EMH) introduced by Fama (1970). EMH consists of three types: the strong form, the semi-strong form, and the weak form. The strong form argues that a market is efficient if all information relevant to the value of a share, whether or not available to existing investors, is reflected in the market price. The semi-strong form holds that a market is efficient if all publicly available information is reflected in the market price. The weak form argues that the current price of an asset reflects all its price and trading volume history. Taking into account this last form of efficiency, it is not possible to develop a price-based investment strategy that beats the market in the mean-variance sense; i.e., such price-based strategy cannot obtain a higher return with equal or less risk than the buy and hold strategy, and such strategy cannot obtain a lower risk with equal or higher return than the buy and hold strategy.

Nonetheless, Malkiel (2003) indicates that future stock prices are at least partially predictable. Criticism of EMH has given rise to an increasing number of papers that question the validity of the EMH basis, and introduce new and successful approaches that combine technical analysis indicators and chart patterns with methodologies from econometrics, statistics, data mining and artificial intelligence. Recent research shows that the combination of some technical analysis such as chart patterns, indicators and oscillators can predict future prices in such a way that profitable strategies can be designed, therefore seriously questioning the fundamentals of EMH (Cervelló-Royo, Guijarro, \& Michniuk, 2015; Gong, Si, Fong, \& Biuk-Aghai, 2016; Leitão, Neves, \& Horta, 2016). Chart patterns are formations created by the movements of prices on a chart. A pattern is usually identified by connecting the price points with a line. Indicators and oscillators are created by applying formulas of varying complexity to historical prices. Oscillators, when used in technical analysis, are a type of technical indicator usually employed for identifying momentum (Teixeira and De Oliveira, 2010). In fact, some papers refer to them as 'momentum oscillators' (Park and Irwin, 2007).

This paper focuses on one of the most analyzed chart patterns in the literature: the flag. Downes and Goodman (1998) defined it as a "technical chart pattern resembling a flag shaped like a parallelogram with masts on either side, showing a consolidation within a trend. It results from price fluctuations within a narrow range, both preceded and followed by sharp rises or declines". The trading rule based on the flag pattern can be considered as a trend following strategy, which assumes that the movement of stock market prices is driven by momentum, i.e., the market price moves in the direction of the momentum (Wu et al., 2016).

Formerly, different pattern definitions of flags were used by Leigh, Modani, Purvis and Roberts (2002), Leigh, Paz and Purvis (2002), Leigh, Purvis and Ragusa (2002) and Wang and Chan $(2007,2009)$. We follow the most recent flag pattern definition by 
Cervelló-Royo et al. (2015) -henceforth CGM- and incorporate some relevant improvements to their trading rule. This enables us to design a more profitable and less risky investment strategy.

We propose to adjust the parameters that define the trading rule in a dynamic way. These parameters are the stop loss and the take profit. We partition the original data into quarters, so that the configuration with the best performance in quarter $q$ is applied on the next quarter $q+1$. The results from CGM are dependent on the parameters, which remain constant for the whole period. Thereby the future investor profit depends on its ability to select the configuration of stop loss and take profit with optimal performance, but, unfortunately, this cannot be known a priori. Our proposal defines the parameters in an objective and automatic way, so the result of the strategy is independent of the investor's expertise. We use each $q$ quarter as a training period, and in accordance with the best profit result then we use the best stop loss and take profit configuration for the next $q+1$ testing quarter.

We also filter each trade by using a very well-known technical indicator: the exponential moving average (EMA). The aim of using the EMA is to filter the bad trades -those that end with losses- by only taking into account those trades in which the flag pattern signal coincides with the indicator signal (both are buy or both are sell signals). The flag is considered by experts as a trend continuation pattern, so adding the EMA indicator does reinforce the continuation signal provided by the flag pattern. The present study thus combines both a classical technical analysis indicator and a chart pattern, which constitutes a difference from CGM.

We simultaneously consider the short and the medium term in the trading signals. The flag pattern is identified on a 15-minute time frame, whereas the EMA is calculated both on 15-minute and daily time frames. Aligning different time frames enables us to analyze both the short and medium term of the price structure and then optimize the investment decision process.

Finally, we filter the trades by only considering 1) "relevant" flag patterns: those with a minimum price range, and 2) by limiting the maximum loss per trade to 100 points, which constrains losses when the flag pattern is too large in comparative terms.

All these enhancements have a positive impact both on return and risk and reduce the number of trades through filtering and, consequently, the cost of transactions. The most important improvement is related to the return/risk ratio, which surpasses the results reported by the previous research. Additionally, our results improve on all the 96 different configurations tested by CGM.

The rest of the paper is structured as follows: Section 2 summarizes the recent literature on trading rules based on technical indicators and trading rules based on chart patterns. Section 3 reviews the flag pattern trading rule. Section 4 introduces the new EMA-flag pattern strategy with the dynamic approach for setting parameters. Section 5 presents the results we obtain when applying the proposal to the intraday data of the Dow Jones Industrial Average (DJIA), and compares them with those reported in CGM and with the buy $\&$ hold strategy. Section 6 explain how data snooping effect can impact the results, and presents its statistical significance through the Reality Check. Finally, the last section summarizes the findings and gives our conclusions. 


\section{Survey of the literature}

This section is devoted to analyzing the most recent contributions to the literature on investment strategies. Firstly, we summarize the ones that make use of technical analysis indicators and then analyze the strategies that are based on chart pattern recognition.

It is worth mentioning that the work of Fang, Jacobsen, and Quin (2014) concludes that simple technical trading rules, i.e., rules based on the consideration of isolated technical indicators such as simple moving averages (SMA), relative strength index (RSI), or moving average convergence/divergence indicator (MACD), have no predictive power on stock markets for out-of-sample periods. This supports our hypothesis that complex and intelligent expert systems need to be developed by efficiently combining chart patterns and technical analysis indicators.

\subsection{Strategies based on technical indicators}

Here we analyze several papers that have recently appeared on the use of technical analysis indicators in stock market price forecasting, combined with some other techniques such as neural networks or support vector machines. A more general review of the literature can be found in Hu, Liu, Zhang, Su, Ngai, and Liu (2015).

An example in the search for alternatives which combine technical indicators and an appropriate tuning of their parameters is the modified version of the moving average proposed by Papailias and Thomakos (2015). The strategy developed by the authors increases the investor's cumulative return and Sharpe ratio while exhibiting a smaller risk as measured by the maximum drawdown and the drawdown duration.

Chang, Liao, Lin, and Fan (2011) use Piecewise Linear Representation (PLR) and Artificial Neural Networks (ANN) to find the best stock turning points based on historical data. These turning points represent short-term trading signals for selling or buying stocks on the market, and are calculated through technical indicators as the moving average (MA) with different lengths, the moving average convergence and divergence (MACD), the relative strength index (RSI), the stochastic line, or the Williams \%R indicator. Turning points serve as input into the ANN to train the connection weights of the model. Then, a dynamic threshold method based on exponential smoothing is applied to determine whether the trading signals obtained from the ANN will trigger a sell or a buy decision.

Luo and Chen (2013) also use PLR to identify the turning points in financial series. However, they argue that ANN has the important disadvantage of over-fitting, so they replace it with support vector machines (SVM). The comparative experiments on 20 shares show a better accuracy and profitability in favor of the PLR-SVM combination.

Instead of applying a generalized model for any financial asset, Chiang, Enke, $\mathrm{Wu}$, and Wang (2016) propose an adaptive stock index trading rule which utilizes an ANN for predicting the future stock index price movement direction. The inputs of the ANN model are the MA and the percentage price oscillator (PPO). The results reported by the authors show a total return of $27.50 \%$ for the trade following signals, and $41.86 \%$ for a denoised version of the strategy, whereas the buy-and-hold only achieve a total return of $12.98 \%$.

Deng and Sakurai (2014) also use several technical indicators for the design of its trading rule: the simple moving average (SMA), the exponential moving average (EMA), the MACD and the RSI. They propose a hybrid approach that combines multiple kernel learning (MKL) with differential evolution (DE). The experimental results on the currency pair EUR/USD showed that trading using the prediction learned by MKL 
yielded consistent profits with a good Sharpe ratio, outperforming other methods in the mean-variance sense.

$\mathrm{Hu}$, Feng, Zhang, Ngai, and Liu (2015) developed the eTrend model, which combines an evolutionary learning method with a trend following strategy. By using classical technical indicators such as MA, MACD and volume, the model finds several profitable trading rules for daily stock market prices, whereas the risk is measured through the Sortino ratio. Kim and Enke (2016) also generated a trading system using trading rules and rough set analysis. The model is divided into three phases, and the technical indicators are considered in the first phase of the model.

Ozturk, Toroslu, and Fidan (2016) use a genetic algorithm (GA) and a greedy search heuristic (GSH) to combine several trading rules based on technical indicators into a single trading rule. The model is tested on the currency pairs EUR/USD and GBP/USD in three different time frames, the results showing an average of $60 \%$ of profitable trades. In a similar way, $\mathrm{Wu}$, Wang, and Chung (2016) argue that using a single trading rule may not be sufficient to predict the stock price trend accurately. To overcome this drawback, they propose the performance-based reward strategy (PRS), which combines different rule parameters for the MA and the range break-out. The best parameter value is determined through time variant particle swarm optimization (TVPSO). They compare the results obtained with PRS to those obtained through econometrical approaches: the random walk, the autoregressive model, and the generalized autoregressive conditional heteroscedasticity (GARCH) model.

Dymova, Sevastianov, \& Bartosiewicz (2010) show some limitations of the combination of fuzzy logic and methods of the Dempster-Shafer theory. They propose an approach to overcome these limitations, and demonstrate its advantages by developing a stock trading expert system optimized and tested on real data from the Warsaw Stock Exchange. Dymova, Sevastianov \& Kaczmarek (2016) propose a Forex trading expert system based on some technical analysis indicators and a new approach to the rule-base evidential reasoning. In the new approach, information on the values of all membership functions representing the intersecting fuzzy classes is preserved and used in the fuzzy logic rules. The advantages of the proposed approach are demonstrated using the developed expert system optimized and tested on real data from the Forex market for the four currency pairs and several intraday time frames.

\subsection{Strategies based on chart patterns}

Next, we summarize some papers which base their investment strategies on chart pattern recognition. As in the previous section, we only concentrate on the most recent papers to appear in the literature by summarizing the main results (for the previous research see the paper from CGM). A detailed explanation of how flags are detected is left for the next section.

Leitão et al. (2016) propose the combination of Perceptually Important Points (PIPs) and Symbolic Aggregate Aproximation (SAX) with GA. PIPs and SAX are used to identify patterns, whereas GA is used to generate investment rules and find optimal solutions. The methodology is applied on daily data from the S\&P500 index, with a return of $76.7 \%$ for 2011-2014 outperforming the buy and hold strategy (61.9\%). However, only 17 trades on average per year are carried out, which may be considered insufficient to conclude significant results. 
Gong, Si, Fong, and Biuk-Aghai (2016) propose extending the subsequence search algorithm UCR Suite with a Support Vector Machine (SVM) to train a classifier for chart pattern-matching. Authors prove how the proposal can be applied to correctly identify typical chart patterns such as the Head\&Shoulders pattern. However, no investment strategy is developed.

The work of Chen and Chen (2016) is focused on flag pattern matching by using PIPs. To promote recognition accuracy, the proposed model uses chart patterns and technical indicators. The methodology is applied to forecasting the NASDAQ and TAIEX indexes, and the results outperform those from the previous research. However, the dataset is composed of daily historical prices, so the number of identified patterns is low.

It is important to note that most of the papers in the literature use daily historical prices, which reduces the number of trades and, consequently, the significance of the results from a statistical point of view. An important problem of overfitting arises when we combine a low number of trades and a complex trading rule, for example, by including a lot of parameters for the pattern definition, or by considering an excessive number of technical analysis indicators.

\section{Flag pattern recognition}

Works by Leigh, Modani, Purvis, and Roberts (2002), Leigh, Paz, and Purvis (2002) and Leigh, Purvis, and Ragusa (2002) deal with flag pattern recognition. To identify the pattern, they used a 10x10 grid of weights which allows matching with the bull flag pattern. However, the determination of the weights can result in a black box for the users (Wang \& Chan, 2007).

Zapranis and Tsinaslanidis (2012) argue that the decision maker has to set specific weights in the template's grid before the identification of a pattern. These weights are set somewhat arbitrarily and the process embeds a level of subjectivity. CGM mitigates this disadvantage by making an alternative choice of weights. Fig. 1 contains the proposed weight grid that allows a bull flag pattern to be identified. In order to identify a movement price as a bull flag pattern, the price should only visit the non-negative cells: i.e., the cells with a grey background. So, the first column in Fig.1 represents the breakout, a sharp increase in the price. Then the price must moderately fluctuate in the upper zone (consolidation).

We can see the grid as a $10 \times 10$ matrix, where the first column corresponds to the price movement in the period $t$, and the last column corresponds to the price movement in the period $t+9$. Note that the construction of the bear flag variation can be easily addressed by pre-multiplying the matrix in Fig. 1 by the anti-identity $10 \times 10$ matrix, so the identification of the flag pattern is easily performed both for the bull and bear variations. Fig. 2 shows this transformation. 
Figure 1. Bull flag template from CGM

\begin{tabular}{|l|l|l|l|l|l|l|l|l|l|}
\hline 0 & 0 & 0 & 0 & 0 & 0 & 0 & 0 & 0 & 0 \\
\hline 0 & 0 & 0 & 0 & 0 & 0 & 0 & 0 & 0 & 0 \\
\hline 0 & 0 & 0 & 0 & 0 & 0 & 0 & 0 & 0 & 0 \\
\hline 0 & 0 & 0 & 0 & -1 & -1 & -1 & -1 & -1 & -1 \\
\hline 0 & 0 & 0 & -1 & -2 & -2 & -2 & -2 & -2 & -2 \\
\hline 0 & 0 & -1 & -3 & -3 & -3 & -3 & -3 & -3 & -3 \\
\hline 0 & -1 & -3 & -5 & -5 & -5 & -5 & -5 & -5 & -5 \\
\hline 0 & -1 & -5 & -5 & -5 & -5 & -5 & -5 & -5 & -5 \\
\hline 0 & -1 & -5 & -5 & -5 & -5 & -5 & -5 & -5 & -5 \\
\hline 5 & -1 & -5 & -5 & -5 & -5 & -5 & -5 & -5 & -5 \\
\hline
\end{tabular}

Figure 2. Bear flag matrix obtained through multiplying the anti-identity matrix by the bull flag matrix

Anti-identity matrix

Bull flag matrix

Bear flag matrix

$\left[\begin{array}{llllllllll}0 & 0 & 0 & 0 & 0 & 0 & 0 & 0 & 0 & 1 \\ 0 & 0 & 0 & 0 & 0 & 0 & 0 & 0 & 1 & 0 \\ 0 & 0 & 0 & 0 & 0 & 0 & 0 & 1 & 0 & 0 \\ 0 & 0 & 0 & 0 & 0 & 0 & 1 & 0 & 0 & 0 \\ 0 & 0 & 0 & 0 & 0 & 1 & 0 & 0 & 0 & 0 \\ 0 & 0 & 0 & 0 & 1 & 0 & 0 & 0 & 0 & 0 \\ 0 & 0 & 0 & 1 & 0 & 0 & 0 & 0 & 0 & 0 \\ 0 & 0 & 1 & 0 & 0 & 0 & 0 & 0 & 0 & 0 \\ 0 & 1 & 0 & 0 & 0 & 0 & 0 & 0 & 0 & 0 \\ 1 & 0 & 0 & 0 & 0 & 0 & 0 & 0 & 0 & 0\end{array}\right]$

$\times\left[\begin{array}{rrrrrrrrrr}0 & 0 & 0 & 0 & 0 & 0 & 0 & 0 & 0 & 0 \\ 0 & 0 & 0 & 0 & 0 & 0 & 0 & 0 & 0 & 0 \\ 0 & 0 & 0 & 0 & 0 & 0 & 0 & 0 & 0 & 0 \\ 0 & 0 & 0 & 0 & -1 & -1 & -1 & -1 & -1 & -1 \\ 0 & 0 & 0 & -1 & -2 & -2 & -2 & -2 & -2 & -2 \\ 0 & 0 & -1 & -3 & -3 & -3 & -3 & -3 & -3 & -3 \\ 0 & -1 & -3 & -5 & -5 & -5 & -5 & -5 & -5 & -5 \\ 0 & -1 & -5 & -5 & -5 & -5 & -5 & -5 & -5 & -5 \\ 0 & -1 & -5 & -5 & -5 & -5 & -5 & -5 & -5 & -5 \\ 5 & -1 & -5 & -5 & -5 & -5 & -5 & -5 & -5 & -5\end{array}\right]$

$\left[\begin{array}{rrrrrrrrrr}5 & -1 & -5 & -5 & -5 & -5 & -5 & -5 & -5 & -5 \\ 0 & -1 & -5 & -5 & -5 & -5 & -5 & -5 & -5 & -5 \\ 0 & -1 & -5 & -5 & -5 & -5 & -5 & -5 & -5 & -5 \\ 0 & -1 & -3 & -5 & -5 & -5 & -5 & -5 & -5 & -5 \\ 0 & 0 & -1 & -3 & -3 & -3 & -3 & -3 & -3 & -3 \\ 0 & 0 & 0 & -1 & -2 & -2 & -2 & -2 & -2 & -2 \\ 0 & 0 & 0 & 0 & -1 & -1 & -1 & -1 & -1 & -1 \\ 0 & 0 & 0 & 0 & 0 & 0 & 0 & 0 & 0 & 0 \\ 0 & 0 & 0 & 0 & 0 & 0 & 0 & 0 & 0 & 0 \\ 0 & 0 & 0 & 0 & 0 & 0 & 0 & 0 & 0 & 0\end{array}\right]$

Potential flag patterns are detected by fitting the template of Fig. 1 over the price window we want to check; thus, the highest price in the window corresponds with the top of the grid, and the lowest price in the window corresponds with the bottom of the grid. The difference between the highest and the lowest value in the price window is called the price range $(R)$.

The goal of this process is to obtain the fit value (FV), which shows the level of matching between the matrix and the price window. Fig. 3 shows an example of bull flag pattern recognition. First, the price window and the grid are superposed. Notice that CGM constrains the price range to the body of the candlesticks, therefore they do not consider the maximum and minimum from the candlestick shadows. Then we observe that all the Japanese candlesticks coincide with 0 value cells, except for the bottom left cell, which has a value of 5. So, the sum of all these overlapped cells is the fit value: $F V=5$. This is the highest fit value we can obtain with the grid weights of Fig. 1. The higher the fit value, the more likely the price window to be recognized as a bull flag pattern. If the price falls 
within the cells with negative weight (the ones with white background), then the fit value is lower and the price window is unlikely to be considered as a bull flag pattern.

Observe that we represent the price by using candlesticks instead of classical lines. The candlestick is much more informative than simple lines, because we can observe the full price evolution in that candlestick, and not only the line connecting the closing prices.

Figure 3. Bull flag pattern recognition on a Japanese candlestick chart. The fit value is obtained by adding the values in matched cells

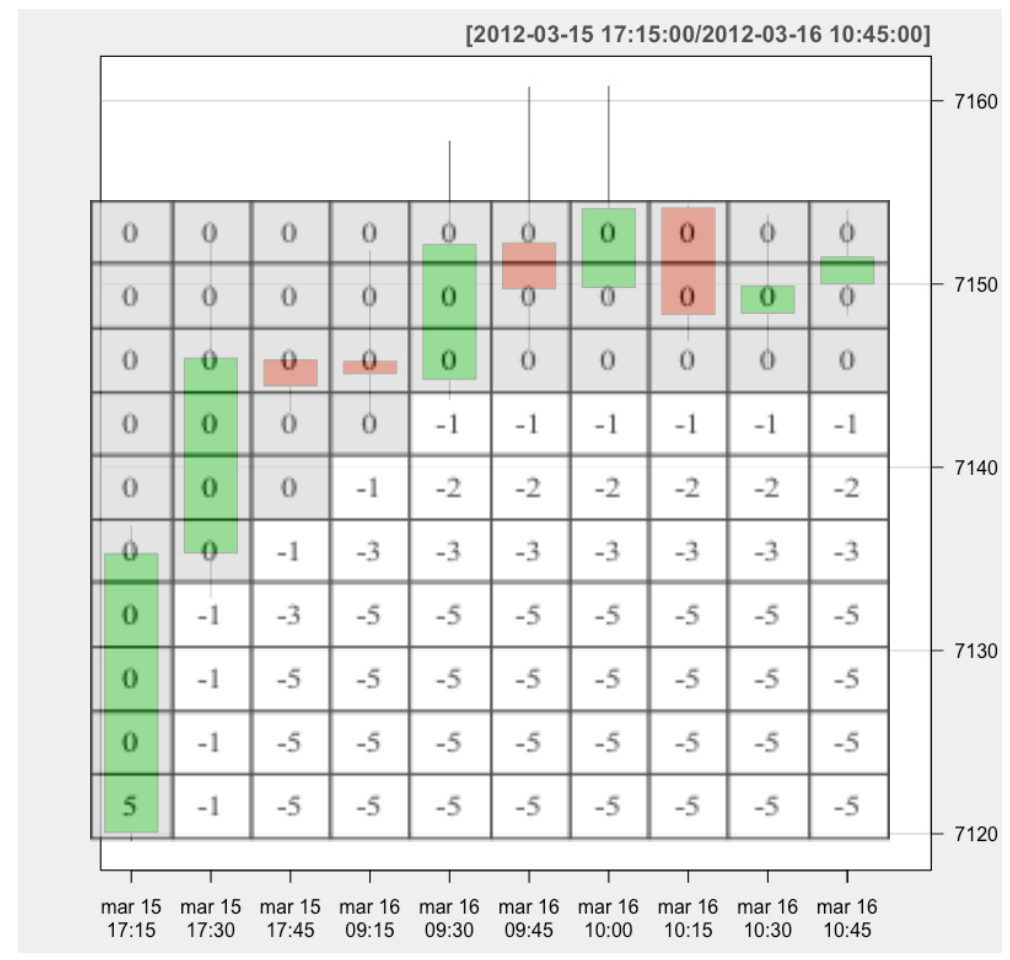

\section{The proposed trading rule}

We now explain the proposed trading rule based on flag pattern recognition. As stated in the Introduction, we use several filters in order to improve its performance.

\subsection{Filtering flag patterns}

The first step is to recognize the bull or bear flag pattern by applying the procedure we explained in the previous section. Notice that the recognition is linked to the fit value (FV). If its value is 5 , we may certainly conclude that the price window contains a flag pattern. Smaller values $(4,3,2$, etc.) remain valid to identify the flag pattern, but the degree of certainty decreases.

Thereby, the fit value constitutes the first parameter to be considered in the strategy, and the performance of the investment depends on the fit value threshold we consider: the smaller the fit value, the more potential flag patterns will be identified and more trades will be executed. However, the accuracy and profitability of these trades will probably also be lower. 
Firstly, we have filtered the flag patterns attending to the price range, by considering only those with a minimum price range of 100 points. This is because some flags are developed within a narrow price range. We argue that the greater the price range, the more significant is the movement for the trader and more confident the trader is in performing the trade. Small movements receive less attention from traders and it is difficult to consider them as an abrupt price rise or fall, so we discard flags with a price range lower than 100 points.

Secondly, we include the momentum effect with a well-known technical analysis indicator: the EMA. When the price is higher (lower) than the EMA, traders consider it as a buy (sell) signal. So, only bull (bear) flag patterns with the price of the last candle higher (lower) than the EMA should be considered as valid flags, whereas the rest are discarded. An important issue is to simultaneously regard both the short and medium term. Thereby, for our intraday trading rule we have computed a short term EMA based on intraday prices (15-minute time frame) and a medium term EMA based on daily prices. The 15-minute EMA was calculated from the closing prices of the previous 50 candlesticks, while the daily EMA was calculated by taking the closing prices of the previous 10 days. In the case of the short-term EMA, a value of $n=50$ implies that the closing prices of the last 12.5 hours, just over a trading day, are being considered. In the case of the medium-term EMA, the evolution of the price in the last 10 days is considered. As it is appropriate to ask how the choice of these parameters affects the results, we analyze this issue at the end of this paper.

A third related filter is considered, which constrains the maximum loss we are willing to have: do not risk more than 100 points in each trade.

Regardless of this maximum permissible loss, the strategy has been parameterized considering different values for the stop loss and the take profit, as detailed in the following section.

\subsection{The take profit and stop loss levels}

Once the flag has been successfully identified, the trade is triggered at the open price of the $11^{\text {th }}$ candlestick. Once opened, we must decide when to close the trade. Most studies consider a holding period of $d$ days (if price time frame is daily): $d \in\{10,20,40,80\}$ in Leigh, Modani, Purvis, and Roberts (2002), $d \in\{20,40,60,80,100\}$ in Leigh, Modani, and Hightower (2004), and $d \in\{20,40,60,80,100,120,160,200,240\}$ in Wang and Chan (2009). Nonetheless, Teixeira and De Oliveira (2010) propose the use of a variation often used by traders: to include a stop loss (SL) and a take profit (TL) in each operation, which will limit both the loss and the profit of trades. This enables the closing of the trade to become dynamic, and not be restricted to a specific number of days or candlesticks, so the duration of each trade is not constant but evolves according to 1) the future movement of price and 2) the price range $R$.

Take profit and stop loss therefore constitute the parameters that define the price level at which the trade must be closed.

Following the bull flag pattern identified on Fig. 3, we put together all the elements that make up the trading rule in Fig. 4. The buy trade is open at the price level of 7152 points. The price range is 34 points (the difference between the highest closing price and the lowest open price). In this example the take profit is placed at 7186 points (the open price plus the 34 points of the range price), whereas the stop loss is set at 7135 points (the open price minus 17 points). So, the take profit is located $1 \times R$ points above the trade open 
price, and the stop loss is $0.5 \times R$ points below the trade open price. Note that these values for the stop loss and the take profit are those used in the example in Figure 4. As we explain below, the work considers a wide range of values to properly capture the sensitivity of the results to the values of these two parameters.

In summary, we make both the take profit and the stop loss dependent on the price range. We follow the common assumption that gains at the take profit level must be considered greater than losses at the stop loss level. This makes the resulting average profit per trade greater than the average loss.

For the validation of the trading rule we have defined the following sensible values in accordance with literature on stop loss and take profit levels:

- $S L \in\{0.2 \times R, 0.3 \times R, 0.4 \times R, 0.5 \times R\}$, with $R=$ price range

- $\quad T P \in\{2 \times S L, 2.25 \times S L, 2.5 \times S L, 2.75 \times S L, 3 \times S L\}$

Therefore, the stop loss goes from 0.2 times the value of the range up to 0.5 times that value, while the take profit has been defined as a function of the stop loss: from 2 to 3 times its value. In total, we have computed $4 \times 5=20$ different configurations for the stop loss and take profit parameters.

Figure 4. An example of triggering a trade entry with take profit and stop loss levels

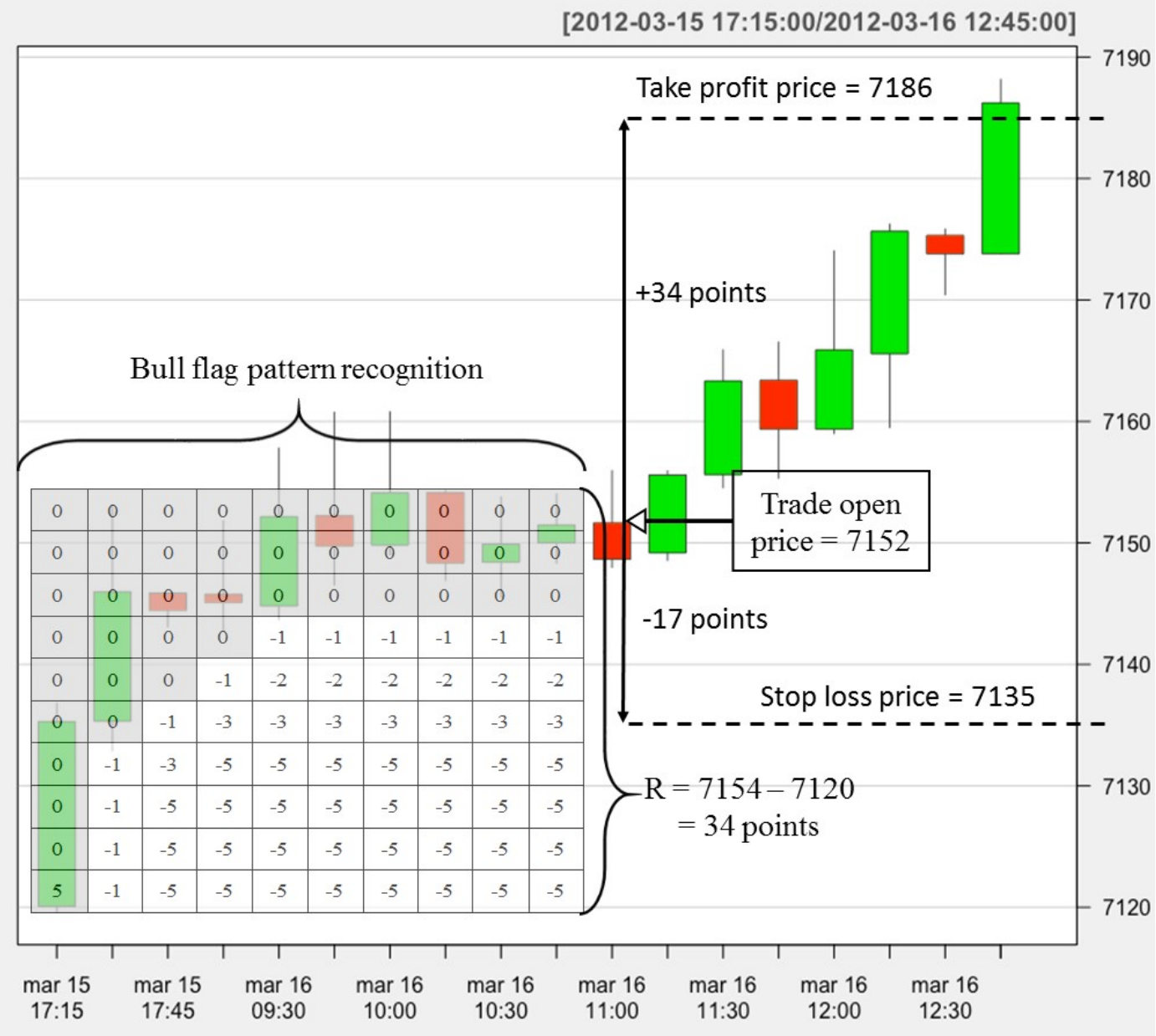


It should be noted that we are supposing that trades occur at the take profit or stop loss trigger prices, but this may not occur in practice. We may encounter slippage problems, so that the trade does not close at the point where we had marked the stop loss, and our loss may be greater than originally anticipated. When the market opens, it may do so at a price which can be different from the one it closed at the previous day, and that open price will cause our loss to be greater than that considered by the stop loss. It may also happen that these price differences benefit us when the price exceeds our take profit. In the case of our work we opted for a conservative approach, considering that in the event of a gap between the close price of a session and the open price of the next session, our loss is the maximum possible (if the price is beyond the stop loss) and our profit is the minimum possible (if the price opens beyond the take profit).

\subsection{The dynamic setting for the take profit and the stop loss}

Once the take profit and stop loss parameters are defined as a multiple of the price range, the following questions arise: what are the optimal values for the take profit and the stop loss? Must these values remain constant, or should they be timely adapted in a dynamic way?

We propose to compute both take profit and stop loss levels in a dynamic manner, taking into account the best recent past performance of the trading rule. We first divide the whole period in quarters $(q)$, and then we compute the best configuration for the take profit and the stop loss for the first quarter $q$. The best configuration for quarter $q$ is then used by the trading rule in the next quarter $q+1$. Therefore, quarter $q$ is used for training whereas quarter $q+1$ is used for testing. Fig. 5 describes the moving window scheme used in our research. The whole period is divided into 53 quarters. Quarters from 1 to 52 are used for training, whereas quarters 2 to 53 are used for testing. Thus, our final results are made up of the profits obtained during the 52 testing quarters. We compute the total profit as the return obtained by all trades executed for the testing quarters 2 to 53 .

Figure 5. Moving window scheme

The dataset

May 22nd 2000 - November 29th 2013

\begin{tabular}{|l|l|l|l|c|c|}
\hline Quarter 1 & Quarter 2 & Quarter 3 & $\ldots$ & Quarter & $\begin{array}{c}\text { Quarter } \\
52\end{array}$ \\
\hline Training & Training & Training & & & \\
\hline
\end{tabular}

We have also measured the risk we have put up with each testing quarter. The nonnormality of the trading rule returns impedes the application of the statistical $t$ (Leigh, 
Modani, and Hightower, 2004). Due to the levels of take profit and stop loss we have chosen, the non-normality of the returns is even higher than in other approaches, and use of the return variance for measuring the risk is not advisable. Instead, we utilize the maximum drawdown as CGM does.

The drawdown at time $t, D_{t}$, is defined in Eq. (1) as the drop of the return curve from the previous maximum at time $s$, with $s<t$. The maximum drawdown $M D$ corresponds to the maximum drawdown experienced during the entire period of the database (Eq. 2).

$$
\begin{gathered}
D_{t}=\max _{s<t}\left(T R_{s}-T R_{t}\right) \\
M D=\max _{t} D_{t}
\end{gathered}
$$

Note that the maximum drawdown refers to the total maximum drawdown, not the maximum drawdown of any particular trade. The maximum drawdown picks the worst run in terms of strategy losses. While these periods can alternate positive trades with negative ones, the maximum drawdown will summarize the combined effect of all of them. The strategy limits the maximum loss for any particular trade to 100 points, with the aim of not being wiped out by a single fatal trade. But this is different from the -totalmaximum drawdown.

Besides, every time a trade closes with losses it does so at the point where it has reached its maximum loss: the stop loss. It is not possible for a trade to close with a loss less than the maximum loss it has been holding while it was open. In other words, it is not possible to have unrealized losses as a result of a trade being in losses and not being closed once the stop loss has been reached.

As a summary of all the above, we have illustrated the process as a whole in Fig. 6. The flag pattern selection process is depicted on the left, while the moving window scheme used for the training and testing the trading rule is summarized on the right.

As a final comment, when applying the strategy to an extensive database it may occur that one trade opens just after another. For example, it is possible to open a long position at a certain time and then open another trade in the same direction in the next candlestick. It is also possible to open a trade while another has not yet been closed, and that both operations may be in opposite directions. The first case assumes that when implementing the strategy on the future of an index, the broker will require a greater margin to keep the trades open. In the other case, the second signal can be implemented by closing the trade in progress from the first signal, and then reopen it when some of the trades are closed by reaching their take profit or stop loss. All these operations are currently available for the most popular trading platforms. 
Figure 6. The flow chart of the proposed trading rule

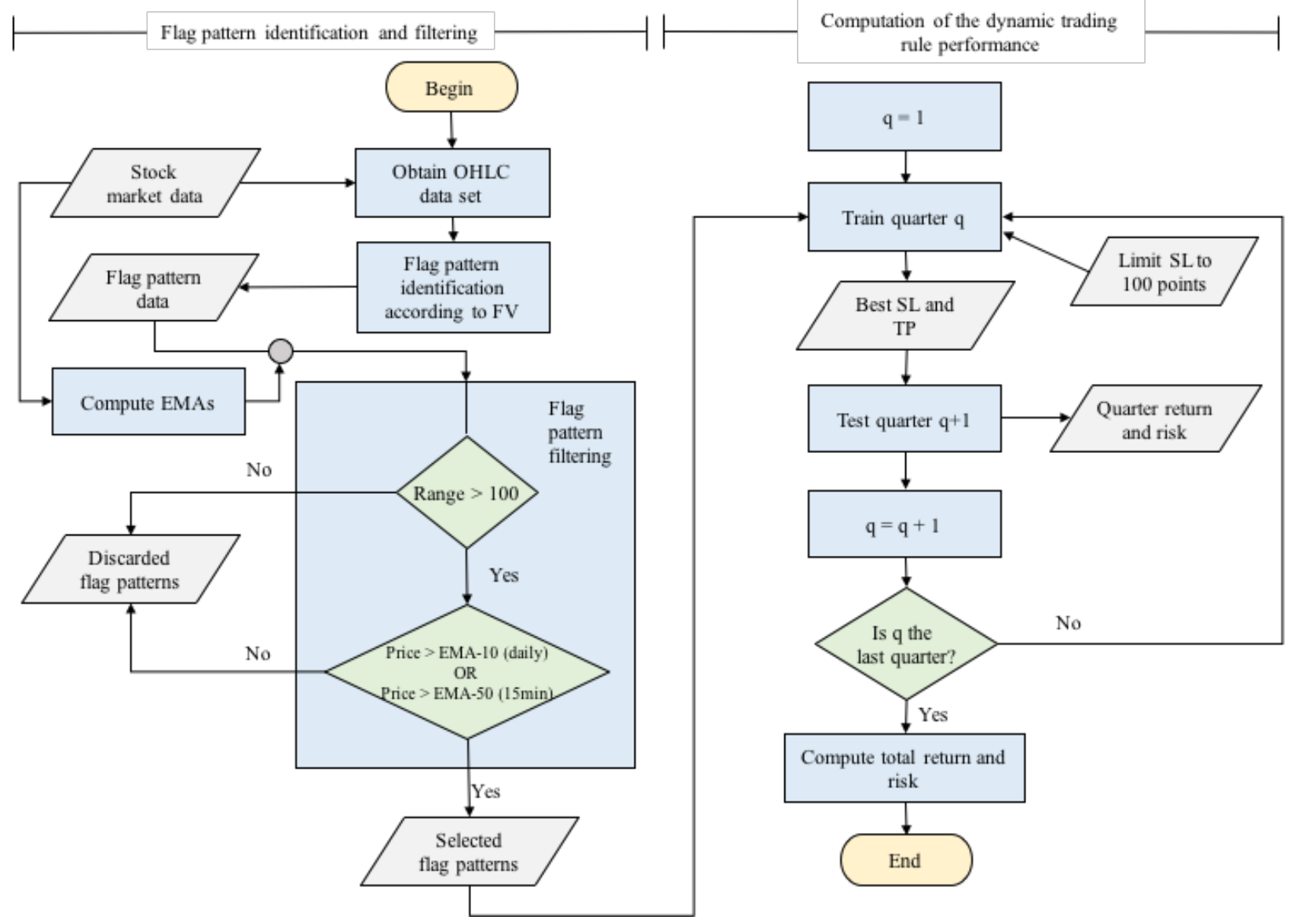

\section{Empirical results and discussion}

To carry out the model comparison under fair conditions, we use the same experimental dataset from CGM: 15-minute candlesticks for the future of Dow Jones Industrial Average index (DJIA) during the period from 22 May 2000 to 29 November 2013, from 9:30 AM to 4:00 PM. Candlesticks with the Open, High, Low and Close prices were directly downloaded from the TradeStation platform. The database has 91,309 candlesticks, and the whole period has been divided into subperiods with 1,750 candlesticks, which approximately corresponds to the periods involved in a quarter.

The use of intraday data has two important advantages over daily data: firstly, we enlarge our dataset and this may entail more trades and more significance from a statistical point of view; secondly, as stated by Schulmeister (2009), the performance of technical analysis has declined over time when based on daily data. However, profitable strategies are found when lower time frequencies -intraday- are used. As in the CGM paper, the use of 5minute or 1 hour data did not generate notable differences from those reported with 15minutes, and for the sake of brevity our work only presents the results in a 15-minutes timeframe.

Following the steps outlined in Figure 6, Table 1 presents the results of applying the methodology proposed on the intraday data of the DJIA index. The results were obtained for a wide range of fit values from +5 to -5 . 
First, we can observe how the number of trades increases in proportion to the fit value. If the flag pattern recognition is done with the maximum fit to the matrix presented in Fig. 1, and in addition we use both the EMA and range filters, then only 293 trades are carried out. This represents a percentage of only $0.3 \%$ of the total candlesticks that make up the sample. To gain statistical significance, we have decided to smooth the fit value requirement to consider values between +5 and -5 . In the latter case, where the fit value is -5 , the number of candlesticks considered is 1,751 , which represents $1.9 \%$ of the sample. Although it may seem that a small proportion of the sample is still represented, we must bear in mind the following: 1) the market does not offer opportunities to buy or sell continuously, and 2) we are only identifying a specific type of opportunity: that of the flag. Other types of chartist figures could be sought so that the number of potential trades could be greater.

Returning to the results of Table 1, we can see that the strategy with the greatest profit is the one that considers a fit value of -5 , with a total of 30,032 points and a return of $286 \%$. However, we could select the best fit value according to the profit obtained by trade. In that case, the best choice would be to take a fit value of +4 , which provides a profit per trade of 26.37 points (return per trade $=0.25 \%$ ).

For the considered fit value range, the lowest profit per trade is given with a fit value of -5 . In general terms, we can see how the profit per trade decreases as the precision in the identification of the flags decreases.

It should be noted that in all cases the profit per trade far exceeds the transaction costs associated with the strategy. There are several financial instruments and derivatives that traders can use for trading and index: futures, options, CFD (contract for differences), ETF (exchange traded funds), etc. If we focus on one of them in particular, for example the case of futures, it is easy to find different brokers and the commissions they apply on the DJIA (E*Trade, Interactive Brokers, OptionXpress, AmeriTrade, TradeStation). The multiplier of a contract for the future E-mini Dow is $\$ 5$. The multiplier is the amount by which the price of this future is multiplied to obtain its monetary value. Therefore, each point of this future has a value of $\$ 5$. At the time of writing, if we buy (sell) an E-mini Dow future when this index is quoted at 20800 points, the monetary value of our trade would be $20800 \times 5=\$ 104,000$.

The most expensive of the brokers cited above would be charging a commission of $\$ 3.50$, i.e. the transaction cost would be $3.50 / 104,000=0.0033 \%$. If we buy the E-mini and the market rises $1 \%$, then we can earn $1 \% \times 104,000=\$ 1,400$, whereas our cost is only $\$ 3.50$. We must also consider the margin the broker requires when we trade. But this is not a cost, because this amount is refunded when we close the trade.

In short, it is possible to trade with the DJIA index through some derivative product, such as a futures contracts. The cost per operation is in many cases less than $\$ 1$ per contract, which takes into account that the $\$ 5$ multiplier that applies to this type of products (Emini Dow future) entails a transaction cost of less than $0.001 \%$ per trade.

Although these transaction costs do not significantly affect the returns obtained by the strategy, we must point out that they are transaction costs for the year 2017. In order to perform an accurate analysis of the profitability of the strategy after commissions, we must know the evolution of transaction costs during the period in which the strategy is implemented. We have completed the analysis by comparing profit and risk. We have reported the maximum drawdown of the strategy according to each fit value and it can be seen that in general terms the risk of the strategy is proportional to the profit. The last column of Table 1 shows the profit in points obtained with respect to the maximum 
drawdown supported. In this case, the best choice would be a fit value of +2 . Again, all the reported cases offer a very favorable risk/reward ratio. In all of them, the profit obtained far exceeds the risk assumed by the strategy.

Table 1. Summary of results according to the fit value

\begin{tabular}{|r|r|r|r|r|r|r|r|}
\hline $\begin{array}{c}\text { Fit } \\
\text { value }\end{array}$ & \multicolumn{1}{c|}{$\begin{array}{c}\text { Number of } \\
\text { trades }\end{array}$} & \multicolumn{1}{c|}{$\begin{array}{c}\text { Profit } \\
\text { (points) }\end{array}$} & \multicolumn{1}{c|}{$\begin{array}{c}\text { Profit per } \\
\text { trade } \\
\text { (points) }\end{array}$} & \multicolumn{1}{c|}{$\begin{array}{c}\text { Profit } \\
\text { (return) }\end{array}$} & \multicolumn{1}{c|}{$\begin{array}{c}\text { Profit per } \\
\text { trade }\end{array}$} & $\begin{array}{c}\text { Maximum } \\
\text { drawdown } \\
\text { (points) }\end{array}$ & \multicolumn{1}{c|}{$\begin{array}{c}\text { Profit / } \\
\text { Maximum } \\
\text { drawdown }\end{array}$} \\
\hline+5 & 293 & 6,559 & 22.39 & $62.5 \%$ & $0.213 \%$ & 707 & 9.28 \\
\hline+4 & 397 & 10,470 & 26.37 & $99.7 \%$ & $0.251 \%$ & 783 & 13.37 \\
\hline+3 & 511 & 12,499 & 24.46 & $119.0 \%$ & $0.233 \%$ & 799 & 15.64 \\
\hline+2 & 626 & 16,454 & 26.28 & $156.7 \%$ & $0.250 \%$ & 1,029 & 15.99 \\
\hline+1 & 933 & 21,097 & 22.61 & $200.9 \%$ & $0.215 \%$ & 1,597 & 13.21 \\
\hline 0 & 1,096 & 22,197 & 20.25 & $211.4 \%$ & $0.193 \%$ & 1,504 & 14.76 \\
\hline-1 & 1,248 & 28,364 & 22.73 & $270.1 \%$ & $0.216 \%$ & 1,894 & 14.97 \\
\hline-2 & 1,393 & 28,165 & 20.22 & $268.2 \%$ & $0.193 \%$ & 1,902 & 14.81 \\
\hline-3 & 1,513 & 29,021 & 19.18 & $276.4 \%$ & $0.183 \%$ & 2,173 & 13.35 \\
\hline-4 & 1,635 & 28,263 & 17.29 & $269.2 \%$ & $0.165 \%$ & 2,609 & 10.83 \\
\hline-5 & 1,751 & 30,032 & 17.15 & $286.0 \%$ & $0.163 \%$ & 2,272 & 13.22 \\
\hline
\end{tabular}

For a more detailed analysis of how the proposed strategy works, Table 2 reports the quarterly results of the trading rule with a fit value of -5 .

The first quarter is used to train the system, after which it is established that the optimal configuration (the one that obtained the highest profit in points) is the one that maintains a stop loss of 0.2 and a take profit of 0.4 in the case of buying trades. For selling trades, the optimal configuration has been a stop loss of 0.2 and a take profit of 0.6 . Therefore, this is the configuration used for the second quarter. We get a profit of 65 points for the buying flags (return $=0.62 \%$ ), while we lose 155 points with selling flags (return $=$ $1.48 \%$ ). This implies that as a whole the strategy loses 90 points in the second quarter.

As with the first quarter, we also get the optimal stop loss and take profit setting for the second quarter: $S L=0.2$ and $T P=0.5$ for the buying side, and $S L=0.2$ and $T P=$ 0.55 for the selling side. This means that the strategy would have optimized its profitability with this configuration, instead of using the quarter 1 optimum. The optimal configuration for quarter 2 is used in the third quarter, gaining 220 points with the long positions, and losing 106 points with the short positions. The accumulated profit up to the third quarter is 285 points (return $=2.71 \%$ ) for long positions and -261 points (return $=$ $2.49 \%$ ) for short positions, bringing the overall profit of the strategy to 24 points.

In this way, the strategy is applied to each of the quarters both for buying and selling opportunities, following the scheme drawn in Fig. 6. The overall result is 1,751 trades, which provide a profit of 14,106 points (return $=134.34 \%$ ) in long trades and a profit of 15,927 (return $=151.69 \%$ ) in short trades. That is, an overall profit of 30,032 points, with the maximum drawdown of only 2,272 points, as pointed out in Table 1 . We have also included the average duration per trade measured by the number of 15-min candlesticks. 
Table 2. Results of applying the proposed methodology on Dow Jones intraday data

\begin{tabular}{|c|c|c|c|c|c|c|c|c|c|c|c|c|c|c|c|c|c|}
\hline & \multicolumn{8}{|c|}{ Buy } & \multicolumn{8}{|c|}{ Sell } & \multirow[b]{2}{*}{$\begin{array}{c}\text { Return } \\
\text { standard } \\
\text { deviation }\end{array}$} \\
\hline Quarter & SL & $\mathrm{TP}$ & $\begin{array}{l}\text { Pos. } \\
\text { trades }\end{array}$ & $\begin{array}{l}\text { Neg. } \\
\text { trades }\end{array}$ & $\begin{array}{c}\text { Acc. } \\
\text { profit } \\
\text { (points) }\end{array}$ & $\begin{array}{l}\text { Acc. } \\
\text { profit } \\
\text { (return) }\end{array}$ & $\begin{array}{c}\text { Return } \\
\text { per trade }\end{array}$ & $\begin{array}{l}\text { Average } \\
\text { duration }\end{array}$ & SL & $\mathrm{TP}$ & $\begin{array}{l}\text { Pos. } \\
\text { trades }\end{array}$ & $\begin{array}{l}\text { Neg. } \\
\text { trades }\end{array}$ & $\begin{array}{c}\text { Acc. } \\
\text { profit } \\
\text { (points) }\end{array}$ & $\begin{array}{c}\text { Acc. } \\
\text { profit } \\
\text { (return) }\end{array}$ & $\begin{array}{c}\text { Return } \\
\text { per trade }\end{array}$ & $\begin{array}{l}\text { Average } \\
\text { duration }\end{array}$ & \\
\hline 2 & 0.2 & 0.4 & 5 & 8 & 65 & $0.62 \%$ & $0.05 \%$ & 10.7 & 0.2 & 0.6 & 0 & 6 & -155 & $-1.48 \%$ & $-0.25 \%$ & 6.9 & $0.09 \%$ \\
\hline 3 & 0.2 & 0.5 & 9 & 15 & 285 & $2.71 \%$ & $0.09 \%$ & 8.9 & 0.2 & 0.55 & 5 & 17 & -261 & $-2.49 \%$ & $-0.05 \%$ & 8.2 & $0.23 \%$ \\
\hline 4 & 0.5 & 1.25 & 2 & 11 & -59 & $-0.56 \%$ & $-0.25 \%$ & 28.3 & 0.5 & 1.5 & 9 & 11 & 1,199 & $11.42 \%$ & $0.70 \%$ & 48.7 & $0.15 \%$ \\
\hline 5 & 0.2 & 0.45 & 7 & 13 & 4 & $0.04 \%$ & $0.03 \%$ & 10.5 & 0.5 & 1.5 & 6 & 13 & 1,379 & $13.13 \%$ & $0.09 \%$ & 38.8 & $0.37 \%$ \\
\hline 6 & 0.5 & 1.375 & 4 & 14 & -253 & $-2.41 \%$ & $-0.14 \%$ & 39.1 & 0.4 & 1.2 & 15 & 23 & 4,270 & $40.67 \%$ & $0.72 \%$ & 29.2 & $0.16 \%$ \\
\hline 7 & 0.2 & 0.5 & 16 & 9 & 568 & $5.41 \%$ & $0.31 \%$ & 17.8 & 0.5 & 1.5 & 2 & 15 & 3,789 & $36.09 \%$ & $-0.27 \%$ & 29.3 & $0.10 \%$ \\
\hline 8 & 0.5 & 1.5 & 7 & 15 & 970 & $9.24 \%$ & $0.17 \%$ & 41.7 & 0.3 & 0.825 & 4 & 13 & 3,663 & $34.89 \%$ & $-0.07 \%$ & 16.4 & $0.13 \%$ \\
\hline 9 & 0.3 & 0.675 & 3 & 21 & 166 & $1.58 \%$ & $-0.32 \%$ & 32.2 & 0.5 & 1.5 & 9 & 9 & 4,570 & $43.52 \%$ & $0.48 \%$ & 30.4 & $0.28 \%$ \\
\hline 10 & 0.2 & 0.45 & 13 & 14 & 826 & $7.87 \%$ & $0.23 \%$ & 12.1 & 0.5 & 1.375 & 15 & 24 & 6,030 & $57.43 \%$ & $0.36 \%$ & 30.9 & $0.33 \%$ \\
\hline 11 & 0.4 & 1.2 & 10 & 28 & 1,235 & $11.76 \%$ & $0.10 \%$ & 25.1 & 0.5 & 1.5 & 7 & 15 & 6,227 & $59.30 \%$ & $0.08 \%$ & 31.5 & $0.21 \%$ \\
\hline 12 & 0.2 & 0.6 & 6 & 13 & 1,389 & $13.23 \%$ & $0.08 \%$ & 37.7 & 0.5 & 1.5 & 7 & 20 & 6,279 & $59.80 \%$ & $0.02 \%$ & 46.7 & $0.14 \%$ \\
\hline 13 & 0.4 & 0.9 & 7 & 10 & 1,731 & $16.49 \%$ & $0.19 \%$ & 26.5 & 0.4 & 1.1 & 0 & 13 & 5,644 & $53.75 \%$ & $-0.47 \%$ & 56.7 & $0.07 \%$ \\
\hline 14 & 0.4 & 0.9 & 0 & 8 & 1,351 & $12.87 \%$ & $-0.45 \%$ & 25.4 & 0.2 & 0.4 & 5 & 2 & 5,823 & $55.46 \%$ & $0.24 \%$ & 13.3 & $0.05 \%$ \\
\hline 15 & 0.2 & 0.6 & 0 & 3 & 1,285 & $12.24 \%$ & $-0.21 \%$ & 31.9 & 0.5 & 1.5 & 2 & 1 & 5,817 & $55.40 \%$ & $-0.02 \%$ & 8.9 & $0.08 \%$ \\
\hline 16 & 0.5 & 1.5 & 2 & 6 & 1,341 & $12.77 \%$ & $0.07 \%$ & 17 & 0.2 & 0.4 & 2 & 1 & 5,888 & $56.08 \%$ & $0.23 \%$ & 7.5 & $0.06 \%$ \\
\hline 17 & 0.4 & 0.8 & 2 & 6 & 1,215 & $11.57 \%$ & $-0.15 \%$ & 71.6 & 0.2 & 0.5 & 1 & 2 & 5,873 & $55.93 \%$ & $-0.05 \%$ & 5.4 & $0.06 \%$ \\
\hline 18 & 0.5 & 1.375 & 3 & 3 & 1,513 & $14.41 \%$ & $0.47 \%$ & 109 & 0.2 & 0.6 & 4 & 0 & 6,138 & $58.46 \%$ & $0.63 \%$ & 13.0 & $0.07 \%$ \\
\hline 19 & 0.5 & 1.5 & 0 & 7 & 1,101 & $10.49 \%$ & $-0.56 \%$ & 39.6 & 0.4 & 0.8 & 0 & 0 & 6,138 & $58.46 \%$ & NA & NA & $0.03 \%$ \\
\hline
\end{tabular}




\begin{tabular}{|c|c|c|c|c|c|c|c|c|c|c|c|c|c|c|c|c|c|}
\hline 20 & 0.2 & 0.6 & 2 & 4 & 1,121 & $10.68 \%$ & $0.03 \%$ & 58 & 0.2 & 0.6 & 0 & 2 & 6,087 & $57.97 \%$ & $-0.25 \%$ & 4.0 & $0.04 \%$ \\
\hline 21 & 0.5 & 1.5 & 1 & 7 & 910 & $8.67 \%$ & $-0.25 \%$ & 29.3 & 0.5 & 1.5 & 0 & 0 & 6,087 & $57.97 \%$ & NA & NA & $0.03 \%$ \\
\hline 22 & 0.2 & 0.6 & 2 & 0 & 1,040 & $9.90 \%$ & $0.62 \%$ & 15.5 & 0.2 & 0.6 & 1 & 1 & 6,130 & $58.38 \%$ & $0.21 \%$ & 1.0 & $0.05 \%$ \\
\hline 23 & 0.5 & 1.375 & 0 & 1 & 968 & $9.22 \%$ & $-0.68 \%$ & 25.4 & 0.5 & 1.5 & 0 & 1 & 6,060 & $57.71 \%$ & $-0.67 \%$ & 8.0 & $0.05 \%$ \\
\hline 24 & 0.5 & 1.25 & 0 & 3 & 788 & $7.50 \%$ & $-0.57 \%$ & 30.1 & 0.2 & 0.55 & 0 & 4 & 5,972 & $56.88 \%$ & $-0.21 \%$ & 108.0 & $0.06 \%$ \\
\hline 25 & 0.3 & 0.9 & 6 & 10 & 1,036 & $9.87 \%$ & $0.15 \%$ & 33.6 & 0.2 & 0.6 & 2 & 3 & 6,047 & $57.59 \%$ & $0.14 \%$ & 10.5 & $0.05 \%$ \\
\hline 26 & 0.5 & 1.5 & 0 & 2 & 926 & $8.82 \%$ & $-0.53 \%$ & 25.2 & 0.3 & 0.675 & 1 & 1 & 6,083 & $57.93 \%$ & $0.17 \%$ & 1.5 & $0.05 \%$ \\
\hline 27 & 0.4 & 1.1 & 0 & 3 & 791 & $7.53 \%$ & $-0.43 \%$ & 15.1 & 0.2 & 0.55 & 2 & 1 & 6,239 & $59.42 \%$ & $0.50 \%$ & 31.0 & $0.09 \%$ \\
\hline 28 & 0.2 & 0.6 & 5 & 10 & 916 & $8.72 \%$ & $0.08 \%$ & 34.9 & 0.5 & 1.5 & 2 & 3 & 6,408 & $61.03 \%$ & $0.32 \%$ & 10.0 & $0.14 \%$ \\
\hline 29 & 0.5 & 1.5 & 9 & 14 & 1,714 & $16.32 \%$ & $0.33 \%$ & 44.6 & 0.3 & 0.75 & 4 & 13 & 6,207 & $59.11 \%$ & $-0.11 \%$ & 23.6 & $0.11 \%$ \\
\hline 30 & 0.5 & 1.125 & 6 & 18 & 1,855 & $17.67 \%$ & $0.06 \%$ & 48.7 & 0.2 & 0.6 & 11 & 9 & 7,206 & $68.63 \%$ & $0.48 \%$ & 12.1 & $0.22 \%$ \\
\hline 31 & 0.4 & 1 & 13 & 25 & 2,375 & $22.62 \%$ & $0.13 \%$ & 45.4 & 0.5 & 1.25 & 25 & 18 & 11,084 & $105.56 \%$ & $0.86 \%$ & 13.5 & $0.19 \%$ \\
\hline 32 & 0.4 & 0.8 & 4 & 18 & 1,758 & $16.74 \%$ & $-0.27 \%$ & 25.8 & 0.5 & 1.375 & 9 & 16 & 12,258 & $116.74 \%$ & $0.45 \%$ & 31.5 & $0.19 \%$ \\
\hline 33 & 0.3 & 0.6 & 12 & 21 & 1,963 & $18.70 \%$ & $0.06 \%$ & 15.3 & 0.5 & 1 & 10 & 25 & 11,944 & $113.75 \%$ & $-0.09 \%$ & 72.2 & $0.35 \%$ \\
\hline 34 & 0.3 & 0.6 & 10 & 19 & 2,266 & $21.58 \%$ & $0.10 \%$ & 11.8 & 0.4 & 1.2 & 16 & 27 & 14,173 & $134.98 \%$ & $0.49 \%$ & 16.4 & $0.43 \%$ \\
\hline 35 & 0.5 & 1.5 & 6 & 21 & 2,236 & $21.30 \%$ & $-0.01 \%$ & 9.1 & 0.5 & 1.5 & 15 & 33 & 14,971 & $142.58 \%$ & $0.16 \%$ & 14.8 & $0.43 \%$ \\
\hline 36 & 0.3 & 0.675 & 10 & 23 & 2,280 & $21.70 \%$ & $-0.58 \%$ & 36.9 & 0.5 & 1.5 & 7 & 18 & 15,043 & $143.27 \%$ & $0.03 \%$ & 31.5 & $0.23 \%$ \\
\hline 37 & 0.2 & 0.5 & 4 & 14 & 2,214 & $21.09 \%$ & $1.05 \%$ & 23.4 & 0.2 & 0.4 & 3 & 11 & 14,904 & $141.94 \%$ & $-0.10 \%$ & 49.1 & $0.06 \%$ \\
\hline 38 & 0.5 & 1.25 & 7 & 16 & 2,409 & $22.94 \%$ & $0.08 \%$ & 13.4 & 0.2 & 0.4 & 7 & 8 & 15,012 & $142.97 \%$ & $0.07 \%$ & 23.7 & $0.14 \%$ \\
\hline 39 & 0.4 & 1.2 & 3 & 6 & 2,423 & $23.08 \%$ & $0.02 \%$ & 72.4 & 0.2 & 0.4 & 4 & 10 & 14,993 & $142.79 \%$ & $-0.01 \%$ & 8.0 & $0.08 \%$ \\
\hline 40 & 0.5 & 1.25 & 9 & 22 & 2,145 & $20.43 \%$ & $-0.09 \%$ & 19.2 & 0.5 & 1 & 9 & 14 & 15,835 & $150.81 \%$ & $0.35 \%$ & 9.3 & $0.24 \%$ \\
\hline 41 & 0.5 & 1.5 & 8 & 11 & 3,172 & $30.21 \%$ & $0.51 \%$ & 30.2 & 0.3 & 0.825 & 4 & 12 & 15,977 & $152.16 \%$ & $0.08 \%$ & 37.7 & $0.13 \%$ \\
\hline 42 & 0.5 & 1.375 & 4 & 11 & 3,289 & $31.32 \%$ & $0.07 \%$ & 71.9 & 0.5 & 1.5 & 3 & 5 & 16,100 & $153.33 \%$ & $0.15 \%$ & 26.7 & $0.14 \%$ \\
\hline 43 & 0.5 & 1.5 & 9 & 3 & 5,248 & $49.98 \%$ & $1.56 \%$ & 120.4 & 0.5 & 1.5 & 2 & 12 & 15,773 & $150.22 \%$ & $-0.22 \%$ & 25.0 & $0.18 \%$ \\
\hline
\end{tabular}




\begin{tabular}{|c|c|c|c|c|c|c|c|c|c|c|c|c|c|c|c|c|c|}
\hline 44 & 0.5 & 1.375 & 9 & 12 & 6,019 & $57.32 \%$ & $0.35 \%$ & 83.3 & 0.3 & 0.9 & 3 & 21 & 15,204 & $144.80 \%$ & $-0.23 \%$ & 27.2 & $0.16 \%$ \\
\hline 45 & 0.5 & 1.5 & 14 & 21 & 9,055 & $86.24 \%$ & $0.83 \%$ & 54.1 & 0.5 & 1.5 & 13 & 29 & 16,835 & $160.33 \%$ & $0.37 \%$ & 19.5 & $0.39 \%$ \\
\hline 46 & 0.5 & 1.375 & 8 & 20 & 9,508 & $90.55 \%$ & $0.15 \%$ & 29.0 & 0.5 & 1.125 & 2 & 24 & 15,398 & $146.65 \%$ & $-0.53 \%$ & 25.9 & $0.26 \%$ \\
\hline 47 & 0.5 & 1.5 & 5 & 12 & 9,499 & $90.47 \%$ & $0.00 \%$ & 65.3 & 0.3 & 0.675 & 3 & 12 & 15,185 & $144.62 \%$ & $-0.14 \%$ & 18.1 & $0.07 \%$ \\
\hline 48 & 0.5 & 1.5 & 8 & 14 & 10,215 & $97.29 \%$ & $0.31 \%$ & 46.1 & 0.5 & 1.25 & 4 & 15 & 14,603 & $139.08 \%$ & $-0.29 \%$ & 20.6 & $0.17 \%$ \\
\hline 49 & 0.4 & 1.2 & 2 & 7 & 10,381 & $98.87 \%$ & $0.18 \%$ & 51.4 & 0.2 & 0.45 & 2 & 8 & 14,406 & $137.20 \%$ & $-0.19 \%$ & 29.7 & $0.14 \%$ \\
\hline 50 & 0.5 & 1.5 & 7 & 9 & 11,724 & $111.66 \%$ & $0.80 \%$ & 115.9 & 0.4 & 1.2 & 1 & 6 & 14,338 & $136.55 \%$ & $-0.09 \%$ & 23.9 & $0.16 \%$ \\
\hline 51 & 0.5 & 1.5 & 5 & 11 & 12,219 & $116.37 \%$ & $0.29 \%$ & 91.9 & 0.3 & 0.9 & 2 & 3 & 14,542 & $138.50 \%$ & $0.39 \%$ & 22.6 & $0.09 \%$ \\
\hline 52 & 0.5 & 1.5 & 9 & 14 & 12,805 & $121.95 \%$ & $0.24 \%$ & 50.0 & 0.3 & 0.9 & 6 & 10 & 15,310 & $145.81 \%$ & $0.46 \%$ & 9.9 & $0.10 \%$ \\
\hline 53 & 0.5 & 1.5 & 9 & 8 & 14,106 & $134.34 \%$ & $0.73 \%$ & 47.4 & 0.4 & 1.1 & 6 & 5 & 15,927 & $151.69 \%$ & $0.53 \%$ & 23.7 & $0.14 \%$ \\
\hline
\end{tabular}

Note: The best configurations are represented separately for the buying and selling trades for each of the tested quarters. Both the stop loss (SL) and the take profit (TP) are defined as a percentage over the range of the flag pattern. We report the number of winning trades (Pos. trades), the number of losing trades (Neg. trades), the total accumulated points (Acc. profit points), the total accumulated return (Acc. profit return), the mean return per trade, the average duration in 15-min candlesticks per trade, and the standard deviation of returns. 
Figure 7 compares the range of the detected flag patterns with the evolution of the DJIA. Again, it is the result obtained with a fit value of -5 . We can see how the flag patterns with the highest range coincide with more abrupt movements of the price in the DJIA, especially when there are big drops in the market. For lower volatility periods, such as that of the 2004-2006 triennium, the range of the flag patterns is smaller on average, and in addition the number of the detected flag patterns is much lower than that observed for the whole period.

Figure 7. A comparison between the range of the detected flag patterns and the DJIA

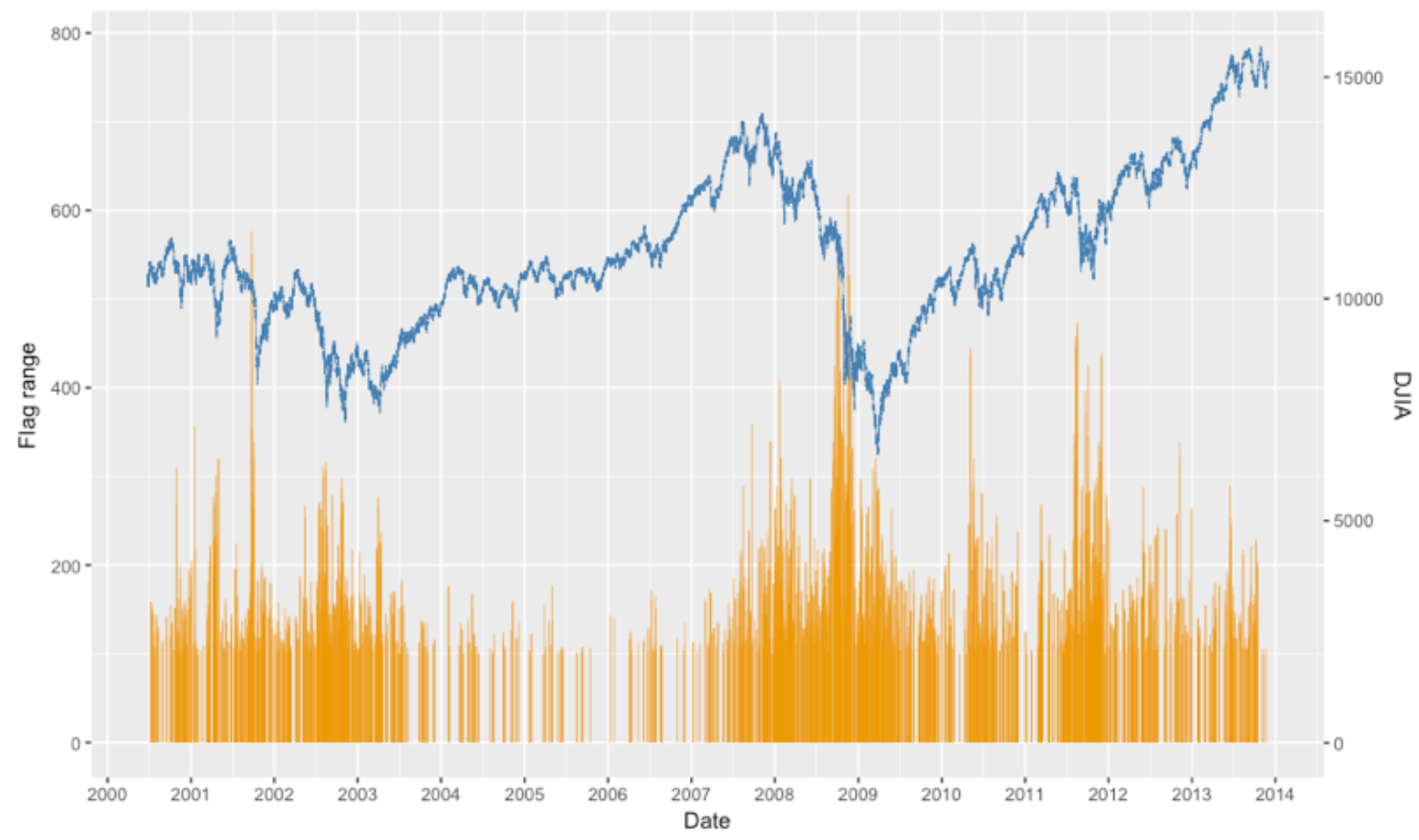

Once these results are obtained, we can ask ourselves if the strategy is simply reacting to the EMA, i. e. to consider if the profit obtained is due to the flags improving the result that the EMA would obtain by itself, or on the other hand, if the EMA improves the results obtained by the flag as a strategy by itself. In order to answer this question, we designed a strategy based solely on the signals offered by the EMA strategy, and compare its results with the CGM flag pattern strategy and those obtained in this work.

The EMA strategy design is simple; if the closing price of a candlestick is above the EMA, then it is considered a buying signal. If it closes below the EMA, the signal indicates selling. In either case, the operation is closed when a new signal is generated in the opposite direction. Following these instructions, as many strategies are generated as parameter $n$ values in the EMA definition. In our case we considered 246 values from $n=5$ to $n=250$.

Figure 8 shows the returns obtained for the 246 different values of the moving average. It can be observed that in the best case the strategy obtains a profit slightly over $200 \%$, while the average profit is $157 \%$. We also represent the number of trades for each EMA strategy. $\operatorname{EMA}(250)$ has the minimum number of trades $(2,362)$ while $\operatorname{EMA}(5)$ has the maximum $(23,781)$. 
The highest profit obtained with CGM is $180.2 \%$, which is less than that obtained by the EMA strategy, and also lower than the one proposed in our work. The average profit for all the CGM configurations is $93 \%$.

To sum up, the EMA strategy obtains a higher mean profit than that obtained with the strategy based on the flag pattern (without taking into account risk or transaction costs), but the combination of both, together with the other elements considered in our work, obtains a higher return than the two previous cases.

Figure 8. Cumulative performance of the EMA-based strategy according to the parameter used for the calculation of the moving average $(n)$

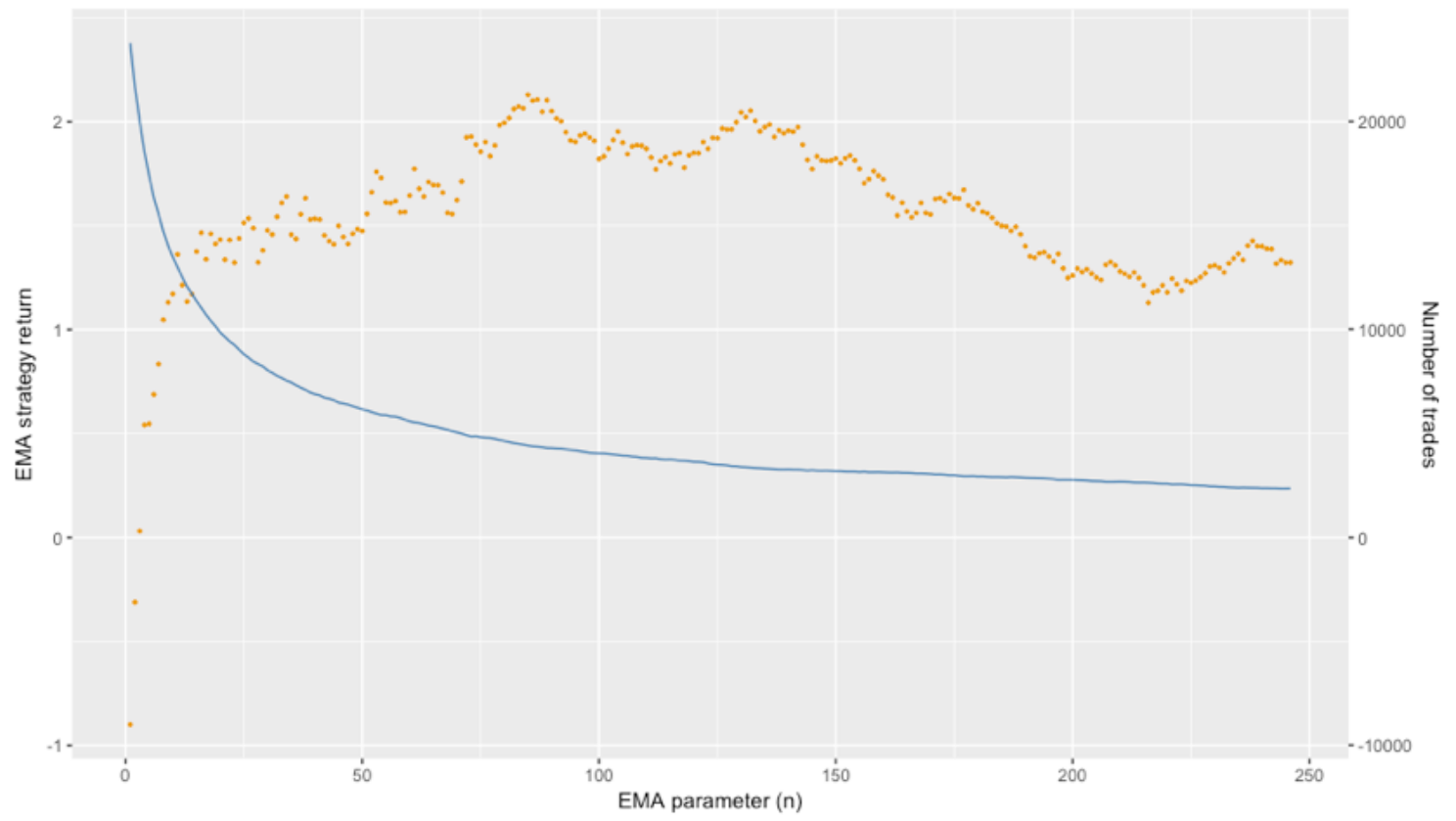

Note: Points account for the EMA strategy return. The curve represents the number of trades executed in each EMA strategy. The $\mathrm{n}$ parameter in EMA goes from $n=5$ to $n=$ 250

It is also interesting to compare the results obtained when applying this strategy with the previous proposal of CGM, or the profit obtained by following a buy \& hold strategy on the DJIA index. Fig. 9 compares the results of applying the proposed strategy in the case of maximizing the number of points obtained (fit value of +5 ), with the best strategy reported in CGM, and the result of following a buy \& hold on the DJIA index.

We can clearly see how our proposal improves on the results of CGM and the buy \& hold strategy. If we stick to profitability, our proposal reaches 30,032 points, while CGM's best alternative gets 17,710 points. Still greater distance exists with the buy \& hold strategy, which for the analyzed period only obtains 5,004 points.

In addition, our proposal gets over 30,000 profit points by making a total of 1,751 trades. The best CGM alternative reaches 17,710 points with 1,576 trades. This means that by making no more than 200 additional trades we get more than 12,000 points ahead of CGM. The difference in profit between the two strategies is considerable, while the transaction costs are similar. Therefore, we conclude that 1) a quarterly review of the stop loss and take profit configuration, as well as 2) filtering the trades by the number of points of the 
flag and by the EMA indicator, and 3) constraining the maximum loss to 100 points, greatly improve the performance of the flag pattern trading rule.

Figure 9. Comparison of the profit obtained with the proposed strategy, compared to the best alternative of CGM and the DJIA

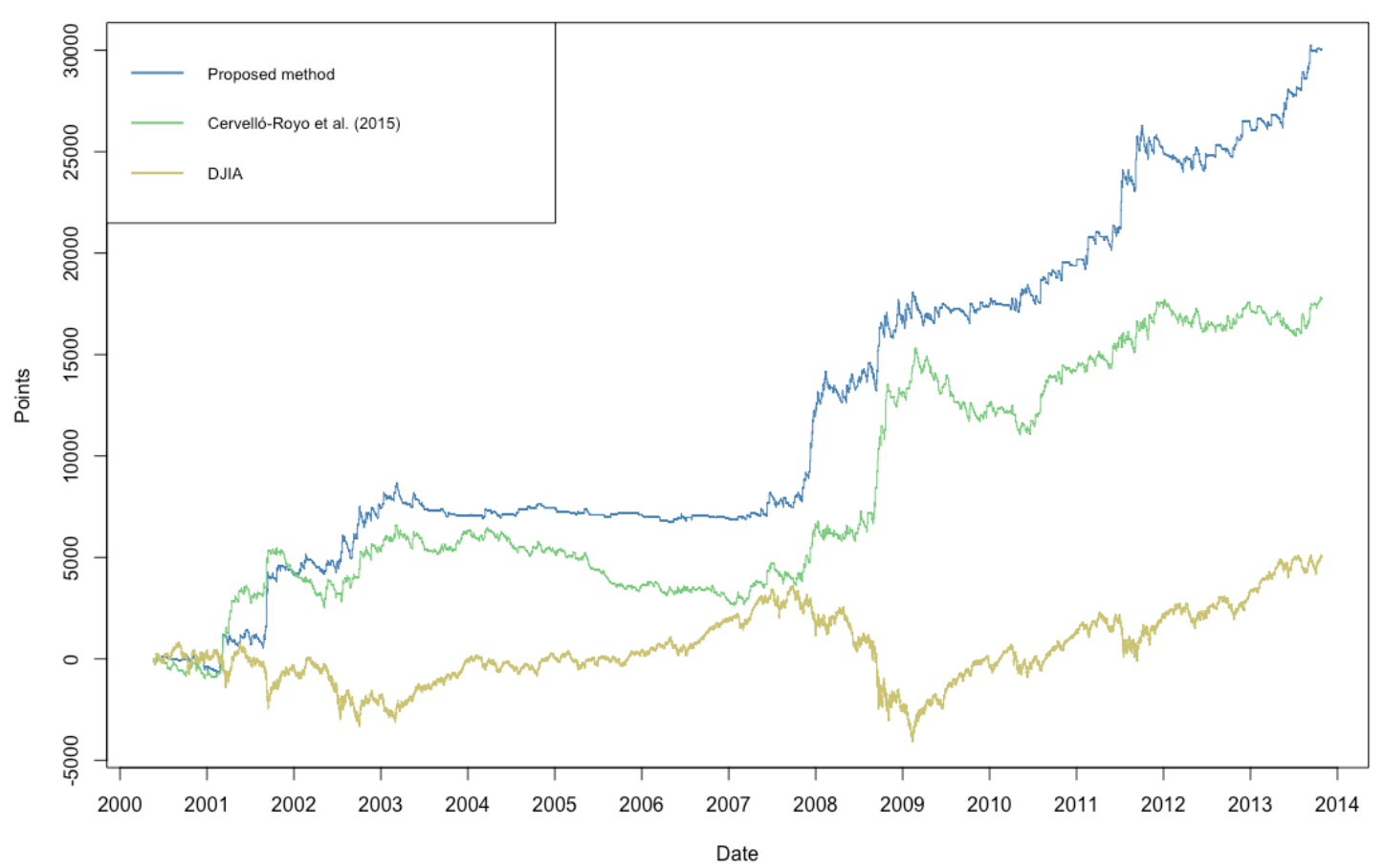

In addition to profitability, our proposal also beats the other alternatives in terms of risk. This risk has been measured through the maximum drawdown. Figs. 10-12 represent the evolution of the drawdown for each of the 3 strategies analyzed. Again, our proposal improves on the results of the other two.

The maximum drawdown reached by our proposal is 2,271 points, while in CGM the best alternative gives a maximum drawdown of 4,244 points. In the worst position is the buy $\&$ hold strategy, with a maximum drawdown of 7,716 points.

In short, we can conclude that our proposal beats the other two in both profitability and risk. 
Figure 10. Evolution of the accumulated profit, the drawdown and the maximum drawdown in the proposed strategy

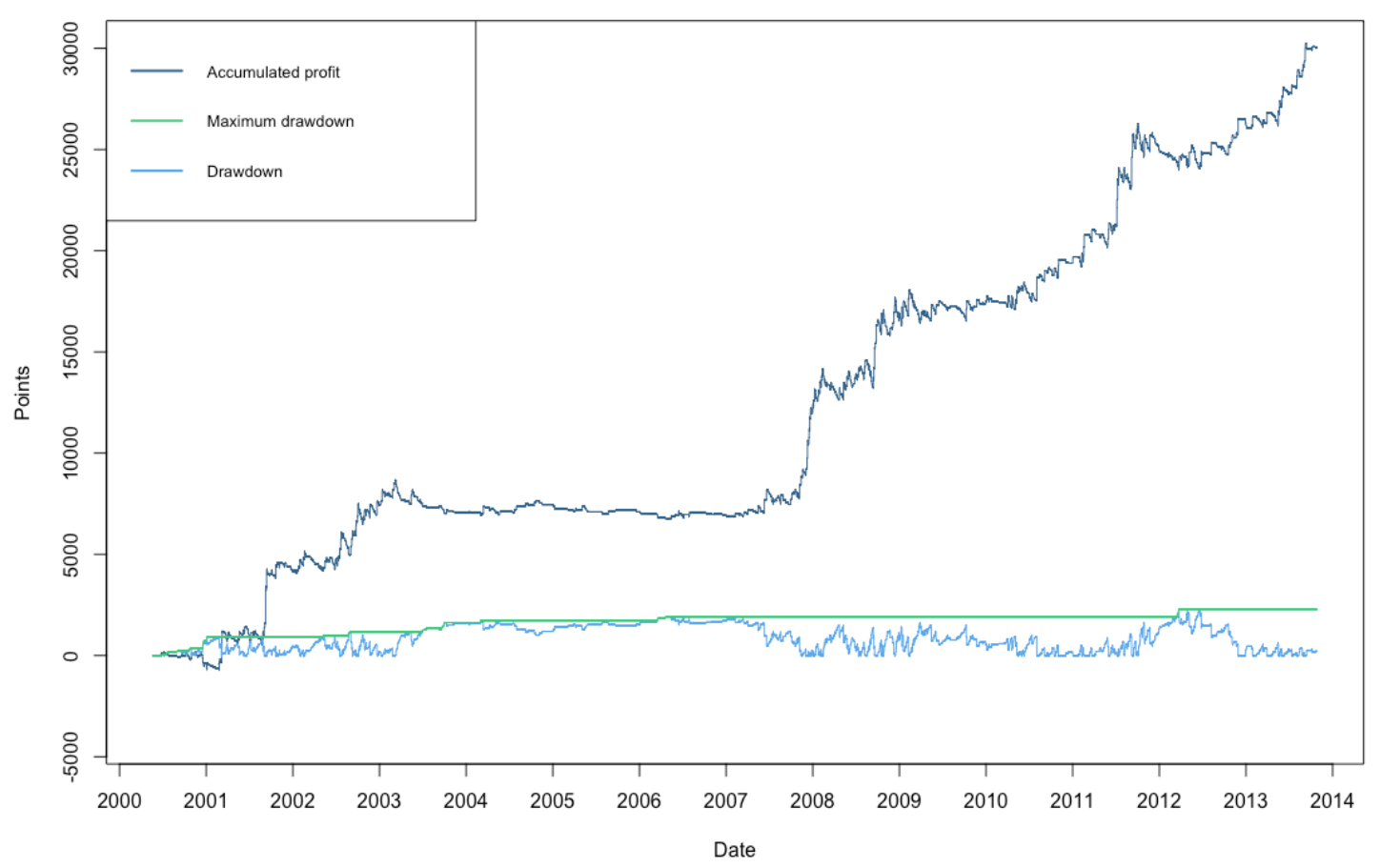

Figure 11. Evolution of the accumulated profit, the drawdown and the maximum drawdown in CGM

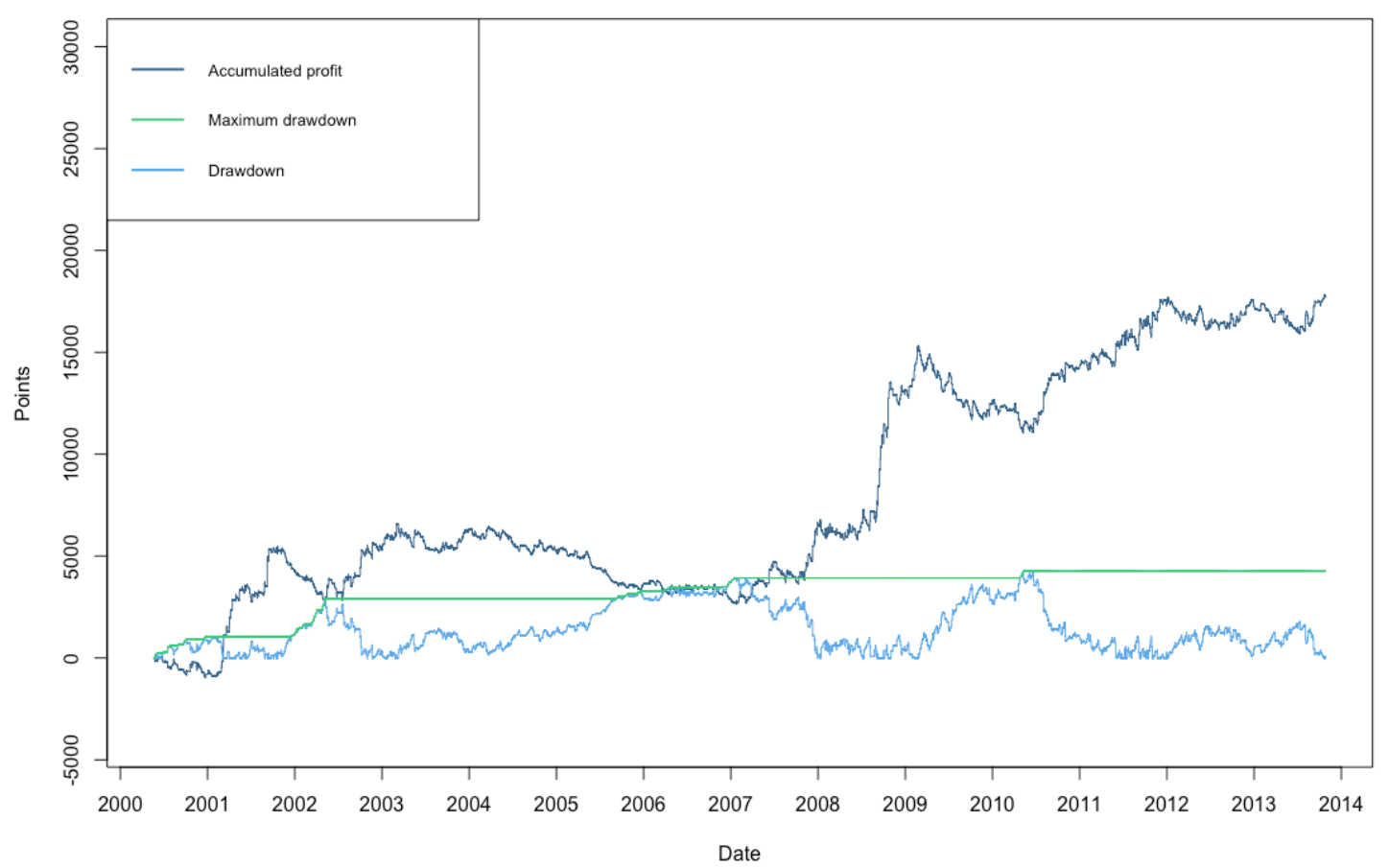


Figure 12. Evolution of the accumulated profit, the drawdown and the maximum drawdown in the buy \& hold strategy

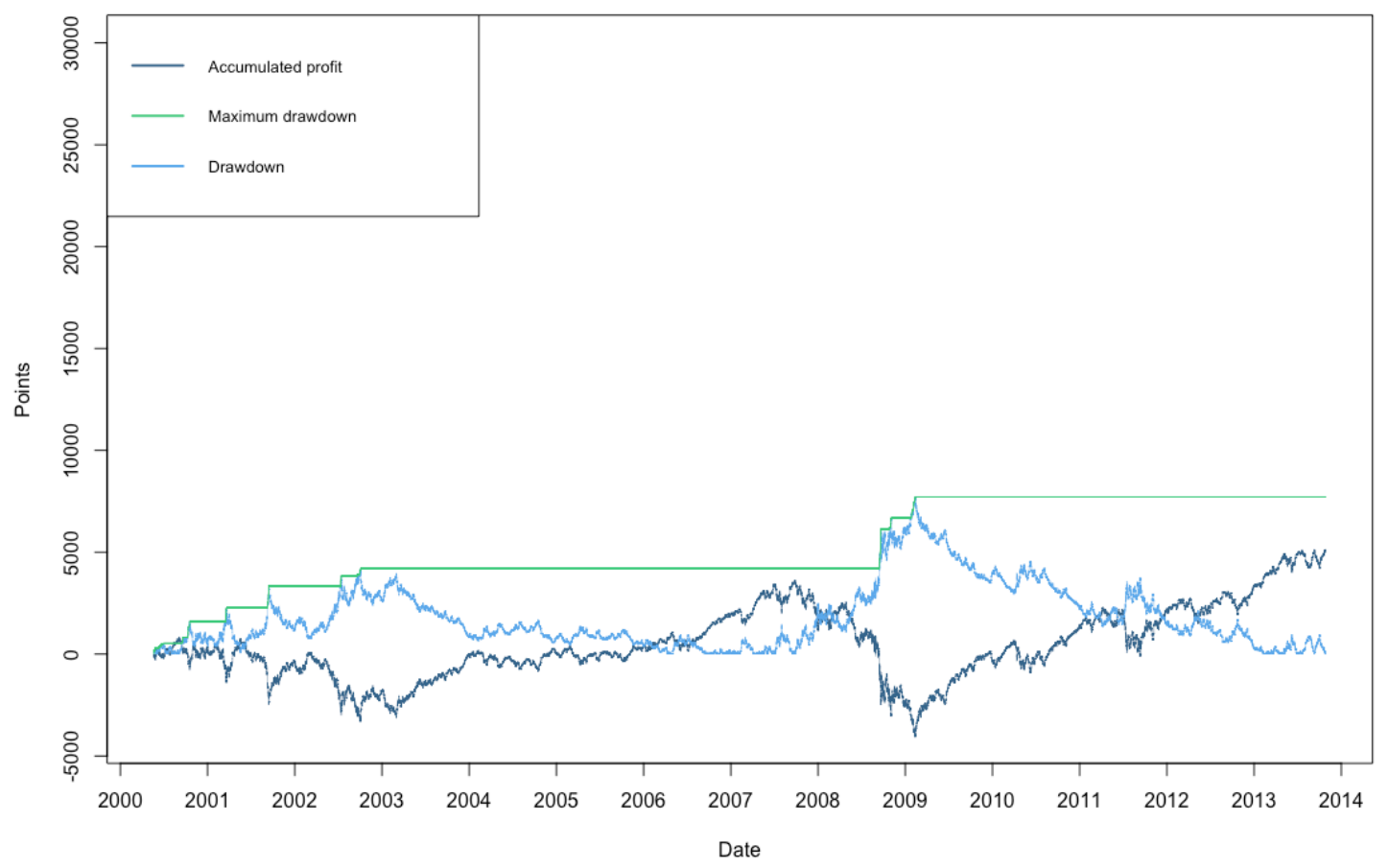

\section{Data snooping test}

Sometimes the effectiveness of trading rules is questioned due to the data snooping effect, which occurs when the same data is used more than once for the purpose of inference or model selection (White, 2000). If the best trading rule (in White's case, model) is obtained through an extensive search for specifications, it is quite possible that this profitable trading rule will be chosen by chance rather than its ability to make a real profit. For example, we can question whether the strategy proposed in our work is profitable only for the parameters chosen for the short-term EMA, medium-term EMA, maximum loss per trade, etc.

While data snooping cannot be completely eliminated, different statistical tests have been developed and applied in the financial field to detect the presence of this effect. White (2000) developed a novel procedure, called the Reality Check, which deals with data snooping biases and allows the calculation of that test. The idea of the Reality Check is to generate the empirical distribution of the complete set of trading rules that leads to the best trading rule, and then extract an inference from this distribution for certain measures of performance.

In a previous study, Brock, Lakonishok, \& LeBaron (1992) find that 26 technical trading rules applied to the DJIA significantly outperform the benchmark of holding cash. Sullivan, Timmermann, \& White (1999) apply the Reality Check by enlarging the database from Brock et al. (1992) and after adjusting for data snooping find that there is insufficient evidence that trading rules have an economic value in predicting the DJIA returns for the most recent 10-year period.

We now describe the procedure to apply the Reality Check to the proposed strategy. 
The procedure starts with the calculation of the $l \times 1$ performance statistic (3):

$$
\bar{f}=n^{-1} \sum_{t=R}^{T} \hat{f}_{t+1}
$$

where $l$ is the number of technical trading rules to be evaluated, $n$ is the number of prediction periods from $R$ through $T$ so that $T=R+n-1$, and $\hat{f}_{t+1}$ is the observed performance measure for period $t+1$ - in our case, the return of the trading rule - .

The null hypothesis to test is that the performance of the best trading rule is no better than the performance of the benchmark (4).

$$
H_{0}=\max _{k=1, \ldots, l}\left\{E\left(f_{k}\right)\right\} \leq 0
$$

where $f_{k, t+1}$ represents the performance of the $k$-th trading rule when compared with the benchmark on day $t+1$ :

$$
f_{k, t+1}=\ln \left(1+r_{t+1} I_{k, t+1}\right)-\ln \left(1+r_{t+1} I_{0, t+1}\right)
$$

$r_{t+1}$ is the return on day $t+1$, defined as $r_{t+1}=\left(p_{t+1}-p_{t}\right) / p_{t} . I_{k, t+1}$ and $I_{0, t+1}$ are the indication of market positions ( +1 long, -1 short, 0 out) on day $t+1$ obtained from the trading signals of the $k$-th trading rule and the benchmark. As stated above, the benchmark can be holding cash, so $I_{0, t+1}=0 \forall t$.

If the null hypothesis (4) is rejected, then we can conclude that the best trading rule is superior to the benchmark (Sullivan et al., 1999). White (1999) evaluates the null hypothesis by applying the Politis \& Romano' stationary bootstrap (1994) to the observed values of $f_{k, t}$. Then $B$ bootstrapped values of $\bar{f}_{k}$ can be obtained by resampling the returns from the $l$ trading rules, denoted as $\bar{f}_{k, i}^{*}$ where $i$ refers to the index of the $B$ bootstrap sample. The following statistics are then calculated:

$$
\begin{gathered}
\bar{V}_{l}=\max _{k=1, \ldots, l}\left\{\sqrt{n}\left(\bar{f}_{k}\right)\right\} \\
\bar{V}_{l, i}=\max _{k=1, \ldots, l}\left\{\sqrt{n}\left(\bar{f}_{k, i}^{*}-\bar{f}_{k}\right)\right\}
\end{gathered}
$$

$\bar{V}_{l}$ is compared to the quantiles of $\bar{V}_{l, i}$ to obtain the Reality Check $p$-value for the null hypothesis (4).

Hansen (2005) noticed that $p$-values increased when poor or irrelevant trading rules produced negative performance measurements, which makes the Reality Check a very conservative procedure. When a trading rule obtains a very poor return, then this translates into a higher value for the $p$-value. even though the rest of the trading rules perform appropriately. Hansen (2005) proposes a more powerful test for Superior 
Predictive Ability (SPA), in which the test statistic takes trading rules with negative return means into account and corrects for potential biases from them.

We have followed the above-mentioned procedure to apply the Reality Check to the proposed strategy. Considering Figure 6, we can see that some parameters are fixed $a$ priori, which may lead one to doubt if these values have been deliberately chosen to achieve an optimal result, or if the strategy would remain profitable for other possible configurations. For the determination of the universe of trading rules we have configured the following parameters:

- Short term EMA (15min): $n=\{35,40,45,50\}$

- Medium term EMA (daily): $n=\{5,7,9,11,15\}$

- Flat pattern's minimum range: $R$ (points) $=\{80,82, \ldots, 110\}$

- Maximum loss per trade: points $=\{90,92, \ldots, 110\}$

Which leads to a total of $l=4 \times 5 \times 16 \times 11=4,224$ trading rules.

It would be possible to include within these parameters the stop loss and the take profit. However, the values for these parameters are not chosen a priori, but are calculated each quarter according to the best result obtained in the previous quarter. Sullivan et al. (1999) state "The cumulative wealth trading rule bases today's signal on the best trading rule as of yesterday, according to total accumulated wealth. The recursive cumulative wealth rule is not the best trading rule ex post, thus the Reality Check $p$-value does not apply". This is why we have not included the stop loss and take profit together with the other parameters mentioned above.

Figure 13 represents the return obtained by each of the 4,224 trading rules generated from the parameters previously mentioned. We can see how you get a positive return, above all in all cases of $240 \%$ and in some cases higher than $300 \%$. The best configuration reported by the CGM hardly obtains a $180 \%$ return, which is the one shown in the figure.

Figure 14 follows a similar one in White (2000), and represents the values of the matrix $V$ for the $l$ trading rules, together with the best relative performance encountered as of the given trading rule.

It is observed that all values are positive, so the $p$-value of the Reality Check is zero. Therefore, we reject the null hypothesis (4) and we can assume that the positive return obtained by the trading rule proposed in the work is not affected by the data snooping effect. SPA, which is not as conservative as the Reality Check, confirms this conclusion. 
Fig. 13. Comparison between the return of the 4,224 trading rules and the best CGM trading rule

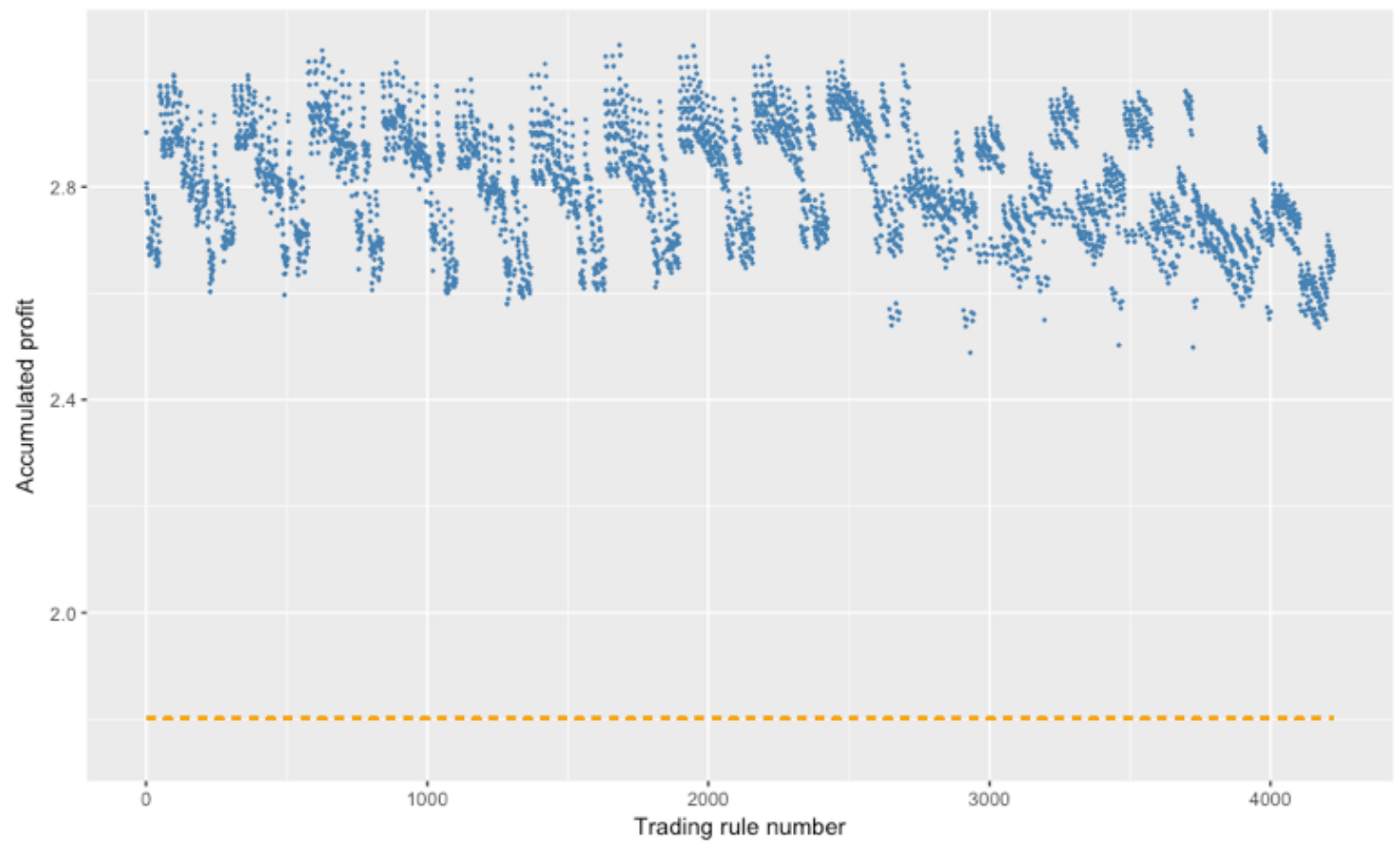

Note: The blue points represent the accumulated return of the $l$ different trading rules. The orange straight dashed line is the accumulated return for the best performance trading rule in CGM.

Fig. 14. The $V$ matrix values from the Reality Check test

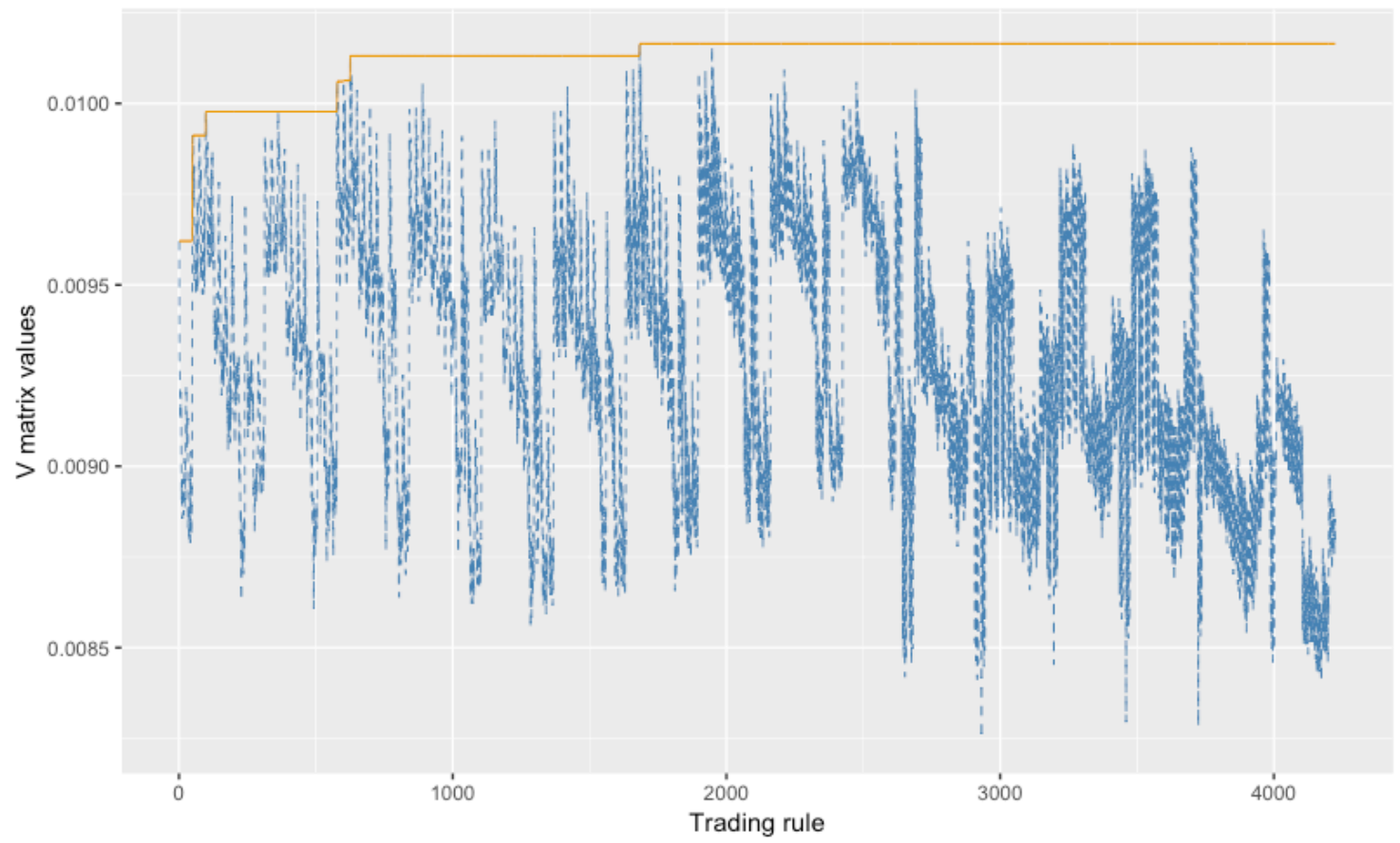

Note: The orange line represents the best relative performance encountered as of the given trading rule. The blue dashed line represents candidate model performance relative to the benchmark. 


\section{Conclusions}

Analysis of the efficiency of stock markets has been the subject of great interest, especially in recent years with the advance in the computation and the treatment of large amounts of information at a great speed. Many recent research studies have detected significant anomalies in the efficiency of markets, some of them linked to chartist patterns. Our work focuses on one of them, the flag pattern.

Following a previous study of ours, Cervelló-Royo, Guijarro, and Michniuk (2015), we have introduced important innovations that have been shown to improve the behavior of the strategy based on the flag pattern in the form of both higher profitability and lower risk. First, the success of the strategy reported in Cervelló-Royo, Guijarro, and Michniuk (2015) depended on how lucky the investor was in choosing the best combination of stop loss and take profit. Our proposal makes this selection automatically, so that these parameters are calibrated in one quarter to be used in the next. In addition, to filter the trades and improve the hit ratio of the strategy, we have introduced the use of the EMA indicator in the short and medium term, which allows only those trades that favor the trend to be launched. We have also discarded those flags that were developed within a very small price range and limited the maximum loss for any operation to 100 points. These innovations allow better selection of the trades, but in a way that the number of trades carried out will continue allowing the results to be statistically significant. The results thus obtained generate a greater profit than the best alternative reported by Cervelló-Royo, Guijarro, and Michniuk (2015) at a lower risk, as measured by the maximum drawdown. In addition, the impact of transaction costs on results is very small, given the high profit per trade obtained with the strategy. In addition, it was also shown that the model does not entail data snooping problems, after generating 4,224 different trading rules and passing the Reality Check test

\section{References}

Barak, S., Dahooie, J. H., \& Tichý, T. (2015). Wrapper ANFIS-ICA method to do stock market timing and feature selection on the basis of Japanese Candlestick. Expert Systems with Applications, 42(23), 9221-9235.

Brock, W., Lakonishok, J., \& LeBaron, B. (1992). Simple technical trading rules and the stochastic properties of stock returns. The Journal of Finance, 47(5), 1731-1764.

Cervelló-Royo, R., Guijarro, F., \& Michniuk, K. (2015). Stock market trading rule based on pattern recognition and technical analysis: forecasting the DJIA index with intraday data. Expert Systems with Applications, 42, 5963-5975.

Chang, P. C., Liao, T. W., Lin, J. J., \& Fan, C. Y. (2011). A dynamic threshold decision system for stock trading signal detection. Applied Soft Computing, 11(5), 3998-4010.

Chen, T. L., \& Chen, F. Y. (2016). An intelligent pattern recognition model for supporting investment decisions in stock market. Information Sciences, 346, 261-274.

Chiang, W. C., Enke, D., Wu, T., \& Wang, R. (2016). An adaptive stock index trading decision support system. Expert Systems with Applications, 59, 195-207.

Deng, S., \& Sakurai, A. (2014). Integrated model of multiple kernel learning and differential evolution for EUR/USD trading. The Scientific World Journal, 2014. 
Downes, J., \& Goodman, J. (1998). Dictionary of finance and investment terms (5th ed.). New York: Barron's Educational Series Inc.

Dymova, L., Sevastianov, P., \& Bartosiewicz, P. (2010). A new approach to the rule-base evidential reasoning: Stock trading expert system application. Expert Systems with Applications, 37(8), 5564-5576.

Dymova, L., Sevastjanov, P., \& Kaczmarek, K. (2016). A Forex trading expert system based on a new approach to the rule-base evidential reasoning. Expert Systems with Applications, 51, 1-13.

Fama, E. F. (1970). Efficient capital markets: A review of theory and empirical work. The Journal of Finance, 25, 383-417.

Fang, J., Jacobsen, B., \& Qin, Y. (2014). Predictability of the simple technical trading rules: An out-of-sample test. Review of Financial Economics, 23(1), 30-45.

Gong, X., Si, Y. W., Fong, S., \& Biuk-Aghai, R. P. (2016). Financial time series pattern matching with extended UCR Suite and Support Vector Machine. Expert Systems with Applications, 55, 284-296.

Hansen, P. R. (2005). A test for superior predictive ability. Journal of Business \& Economic Statistics, 23(4), 365-380.

Hu, Y., Feng, B., Zhang, X., Ngai, E. W. T., \& Liu, M. (2015). Stock trading rule discovery with an evolutionary trend following model. Expert Systems with Applications, $42(1), 212-222$.

Hu, Y., Liu, K., Zhang, X., Su, L., Ngai, E. W. T., \& Liu, M. (2015). Application of evolutionary computation for rule discovery in stock algorithmic trading: A literature review. Applied Soft Computing, 36, 534-551.

Kim, Y., \& Enke, D. (2016). Developing a rule change trading system for the futures market using rough set analysis. Expert Systems with Applications, 59, 165-173.

Leigh, W., Modani, N., \& Hightower, R. (2004). A computational implementation of stock charting: Abrupt volume increase as signal for movement in New York stock exchange composite index. Decision Support Systems, 37, 515-530

Leigh, W., Modani, N., Purvis, R., \& Roberts, T. (2002). Stock market trading rule discovery using technical charting heuristics. Expert Systems with Applications, 23, 155159.

Leigh, W., Paz, N., \& Purvis, R. (2002). Market timing: A test of a charting heuristic. Economics Letters, 77, 55-63.

Leigh, W., Purvis, R., \& Ragusa, J. (2002). Forecasting the NYSE composite index with technical analysis, pattern recognizer, neural network, and genetic algorithm: A case study in romantic decision support. Decision Support Systems, 32, 361-377.

Leitão, J., Neves, R. F., \& Horta, N. (2016). Combining rules between PIPs and SAX to identify patterns in financial markets. Expert Systems with Applications, 65, 242-254.

Luo, L., \& Chen, X. (2013). Integrating piecewise linear representation and weighted support vector machine for stock trading signal prediction. Applied Soft Computing, 13(2), 806-816.

Malkiel, Burton G. (2003). The efficient market hypothesis and its critics. The Journal of Economic Perspectives, 17(1), 59-82. 
Ozturk, M., Toroslu, I. H., \& Fidan, G. (2016). Heuristic based trading system on Forex data using technical indicator rules. Applied Soft Computing, 43, 170-186.

Papailias, F., \& Thomakos, D. D. (2015). An improved moving average technical trading rule. Physica A: Statistical Mechanics and its Applications, 428, 458-469.

Park, C. H., \& Irwin, S. H. (2007). What do we know about the profitability of technical analysis? Journal of Economic Surveys, 21(4), 786-826.

Politis, D. N., \& Romano, J. P. (1994). The stationary bootstrap. Journal of the American Statistical Association, 89(428), 1303-1313.

Schulmeister, S. (2009). Profitability of technical stock trading: Has it moved from daily to intraday data? Review of Financial Economics, 18(4), 190-201.

Sullivan, R., Timmermann, A., \& White, H. (1999). Data-snooping, technical trading rule performance, and the bootstrap. The Journal of Finance, 54(5), 1647-1691.

Teixeira, L., \& De Oliveira, A. (2010). A method for automatic stock trading combining technical analysis and nearest neighbor classification. Expert Systems with Applications, 37, 6885-6890.

Wang, J., \& Chan, S. (2007). Stock market trading rule discovery using pattern recognition and technical analysis. Expert Systems with Applications, 33, 304-315.

Wang, J., \& Chan, S. (2009). Trading rule discovery in the US stock market: An empirical study. Expert Systems with Applications, 36, 5450-5455.

Wang, F., Philip, L. H., \& Cheung, D. W. (2014). Combining technical trading rules using particle swarm optimization. Expert Systems with Applications, 41(6), 3016-3026.

White, H. (2000). A reality check for data snooping. Econometrica, 68(5), 1097-1126.

Wu, M. E., Wang, C. H., \& Chung, W. H. (2016). Using trading mechanisms to investigate large futures data and their implications to market trends. Soft Computing, 114. DOI: $10.1007 / \mathrm{s} 00500-016-2162-6$.

Zapranis, A., \& Tsinaslanidis, P. E. (2012). A novel, rule-based technical pattern identification mechanism: Identifying and evaluating saucers and resistant levels in the US stock market. Expert Systems with Applications, 39(7), 6301-6308. 